Aus der kaiserl. chirurgischen Universitätsklinik Kyoto, Japan (Prof. H. Ito).

\title{
Experimentelle und literarische Studien über die ausgedehnten Dünndarmresektionen.
}

\author{
Von Assistent-Prof. Y. Soyesima.
}

Wieviel Darm kann der Mensch wohl entbehren? Darauf ist bisher sehr verschiedenerweise geantwortet.

B a um behauptete im Jahre 1884 , da $B$ die Dünndarmresektion ron $137 \mathrm{~cm}$ schon weit hinauf die Zulässigkeitsgrenze überschreite.

15 Jahre darnach äußerte sich darüber D reesmann, gestützt auf seinen eigenen Fall und 25 weitere Fälle von Darmresektionen über $1 \mathrm{~m}$ aus der Literatur, wie folgt: ..Wir sehen hieraus, daß Resektionen von etwa über $2 \mathrm{~m}$ anscheinend nur von jugendlichen Personen ohne spätere Störungen ertragen werden. Resektionen von unter $2 \mathrm{~m}$ rufen hingegen keine Verdauungsstörungen hervor; nur wenn Komplikationen vorliegen, können dieselben eintreten und sogar schließlich den Tod herbeiführen."

Nach $\mathrm{Sch}$ lat ter sollen selbst bei einer nur $1^{1}$. $\mathrm{m}$ betragenden Darmkürzung funktionelle Störungen auftreten.

Im Jahre igor führte Albu Stoffwechseluntersuchungen am 4rjährigen Patienten Lexers mit $2 \mathrm{~m}$ Dünndarmresektion wegen Mesenterialfibroms $\mathrm{I}^{1}$, Jahre nach der Operation aus und kam zu clem Schlusse, daß mehr als cin Drittel der im Verhältnis zur Körpergröße berechneten Länge des Dünndarms nirht ohne Getährdung des Ernährungszustandes entfernt werden darf.

Lauwers ist der Ansicht, dal3 man ungestraft die Hälfte des Dünndarms resezieren kann, besonders wenn es sich um Ileum handele.

Auch Kukula sagt, daß Dünndarmresektion bis zur Hälfte seiner Länge beim Menschen ein vollkommen zulässiger und berechtigter Eingriff sei.

$S$ t or p resezierte bei cinem 21 jährigen Manne ein $510 \mathrm{~cm}$ langes Stück von Dünndarm wegen eines Sarkoms aus der Mesenterialwurzel mit Erfolg und glaubt, daB bei normaler Beschaffenheit des zurückbleibenden Darms, relativ ausgedehnte Kürzungen bis zu $2 / 3$ 
und sogar noch darüber ohne bleibenden Nachteil vom menschlichen Organismus vertragen werden können.

Nach Axhausen bilde eine Kürzung des Dünndarms um 80 Prozent entweder die Grenze oder dürfte doch der Grenze nahe stehen, bis zu welcher Dünndarmkürzungen ohne dauernde Beeinträchtigung des Ernährungszustandes möglich seien.

Auch die Ergebnisse der Tierexperimente stimmen miteinander gar nicht überein wie die oben zitierten klinischen Erfahrungen.

Im Jahre 1887 sprach sich Sen $\mathrm{n}$ im 9. internationalen medizinischen Kongreß zu Washington folgendermaßen aus: „As an approximate estimate the statement can be ventured that in dogs and cats the excision of more than one-third of length of the small intestines is dangerous to life, as it is followed by marasmus, which sooner or later results in death."

Später führte Trzebicky an 28 Hunden kürzere und längere Resektionen an verschiedenen Stellen des Dünndarms aus und kam zu dem Schlusse: „Daß Resektionen des halben Dünndarms (Duodenum nicht mitgerechnet) noch ganz gut vertragen werden. Erst venn die resezierte Partie mehr als die Hälfte des Jejunum und Ileum betrügt, wird der Zustand gefährlich, obwohl auch noch hier bei zweckmäßiger Linnährung und sonst normaler Gesundheit das I.eben manchmal erhalten werden kann. Resektionen von $2 / 3$ des Jejunum und Ileums und darüber brechen in den Verdauungs-Chemismus und Mechanismus cine derartige Bresche, daß eine Erhaltung des Lebens unmöglich ist."

Nach $\mathrm{N}$ ag a no vermag der Hund die Resektion von mehr als $2 / 3$ des ganzen Dünndarms ohne Schaden nicht dauernd zu überstehen.

Takay as u exkludierte an 33 Hunden den Dünn- und Dickdarm in verschiedenen Ausdehnungen. Die Ergebnisse seiner Experimente sind kurz folgende: Die maximale Grenze der Ausschaltung betrage beim Dünndarm $2 / 3$ der ganzen Länge (das Duodenum nicht mitgerechnet). Die Ausschaltung des halben Dünndarms dürfe als gefahrlos erachtet werden. Dagegen sei die Ausschaltung bzw. Resektion von $9 / 10$ des Dünndarms oder vom halben Dickdarme samt $3 / 4$ des Dünndarms in allen Fällen mit Gefahr verbunden, indem die Tiere nach einer geraumen Zeit an Ernährungsstörung zugrunde gehen sollen. Er konnte nur einmal an einem kräftigen Hunde $9 / 10$ des Dünndarms mit Erfolg ausschalten.

Diliberti-Herbin sagt, daB die operative Ausschaltung funktionstüchtigen Dünndarms bei Hunden bis zur Hälfte der Gesamtlänge ohne irgend einen Schaden für das Leben der Tiere ausführbar sei. 
Evans und B renizer resezierten an Hunden bis zu 84,6 Proz. der ganzen Länge des Dünndarms mit Erfolg, während die Entfernung desselben um 86,5, 87,3 bzw. 92 Proz. die Tiere später zum Exitus führte.

London und Dmitriew experimentierten an 3 Hunden und kamen zu dem Schlusse: „Eine Resektion von $i_{/}$des Dündarmes kann der Hund im allgemeinen ertragen. Die Ausschaltung des ganzen Dïnndarms beim Hunde von der Plica duodenojejunalis bis zur Valvula Bauhini richtet das Tier schließlich zugrunde."

Mo na r i behauptet, daß die Resektion von $i / s$ des Dünndarms vom Hunde ertragen werde, daB bei Resektion von $\% / 9$ und $9 / 10$ desselben Abmagerung infolge von Diarrhöen eintrete und daß bei noch größeren Resektionen der Exitus erfolge.

Es ist wohl denkbar, daB die Verdauung und Resorption der Nahrung nach den ausgedehnten Resektionen des Dünndarms mehr oder weniger gestört werden. Die Resultate der bis jetzt veröffentlichten Untersuchungen stellen wir in einer Tabelle $z \mathrm{u}-$ sammen (siehe Tabelle S. 428--43I).

Überblicken wir die oben angeführten klinischen Erfahrun. gen und experimentellen Ergebnisse, so finden wir ohne weiteres, daß sie unter sich und miteinander gar nicht übereinstimmen. Dies kommt meines Erachtens vor allem daher, daß die klinisch gut beobachteten Fälle noch nicht zahlreich genug sind, um das letzte Urteil fällen zu können. Dazu ist der Zustand des Patienten und die Natur der Krankheit je nach den einzelnen Fällen sehr verschieden. Es ist selbstverständlich, daß die Prognose der Operation bei den sonst gesunden kräftigen Patienten im besten Mannesalter viel besser ist als bei den schon weit heruntergekommenen Personen im Greisen- oder Kindesalter.

Was das Alter anbelangt, so kann rach $S$ torp bei jüngeren Individuen die funktionelle Anpassung des Darmtraktus nach ausgedehnter Resektion relativ rasch zustande kommen. Hingegen fand Flint experimentell, daß ,Young growing animals apparently do not stand the operation for the removal of large amount of the intestine as well as older dogs".

Ferner hat die individuelle Verschiedenheit der menschlichen Darmlänge dabei sehr viel zu sagen. Bekanntlich ist die Darmlänge der Körper- bzw. Rumpflänge nicht regelmäßig 


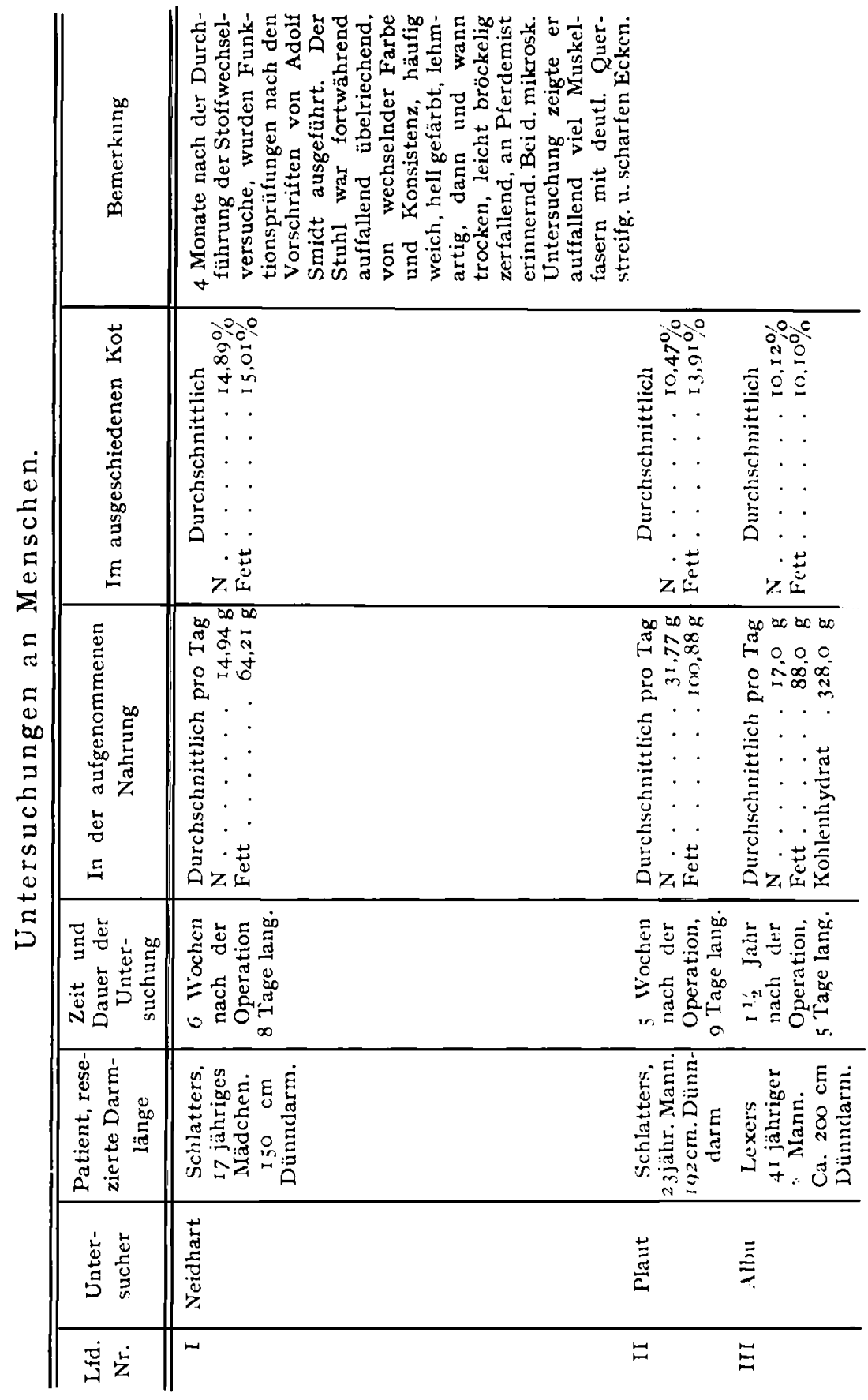




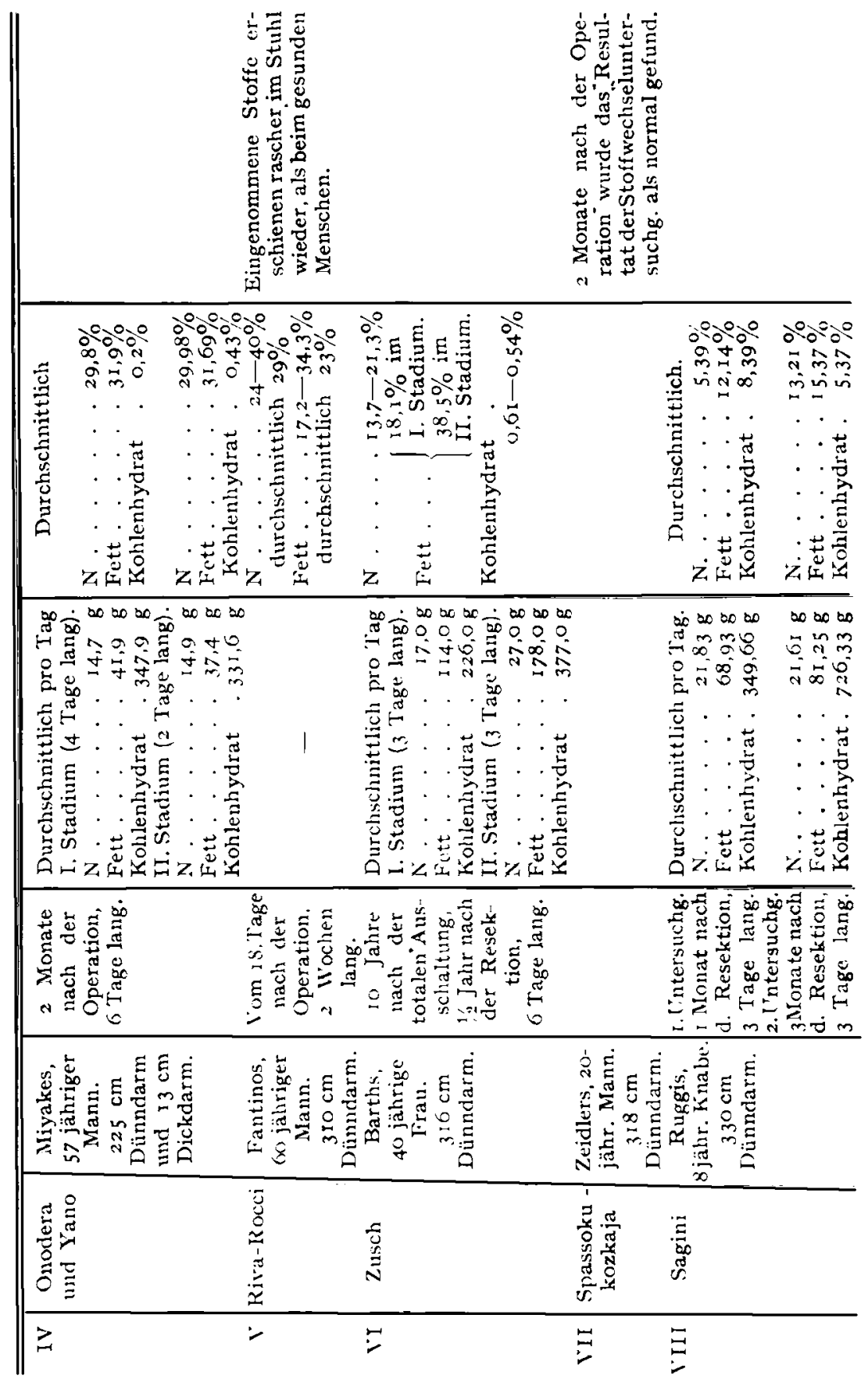




\begin{tabular}{|c|c|c|c|c|}
\hline 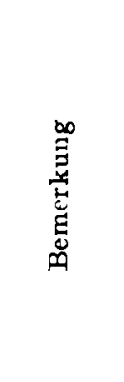 & 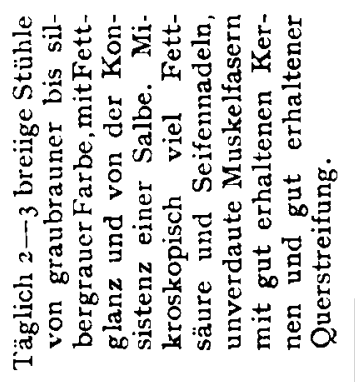 & 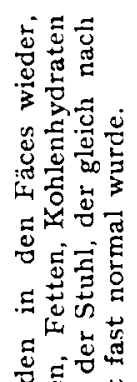 & 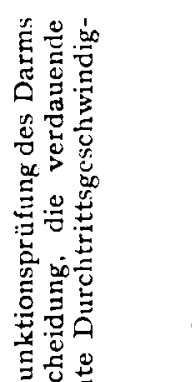 & 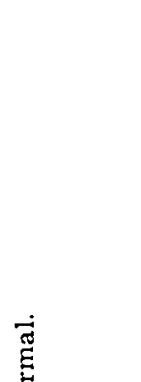 \\
\hline 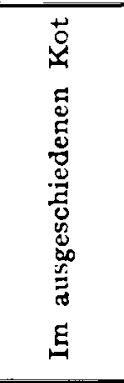 & 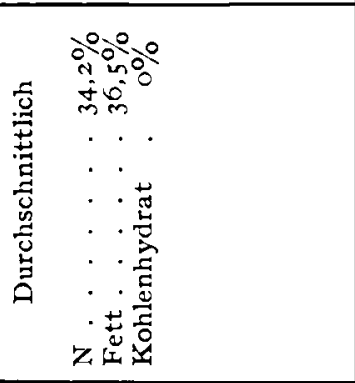 & 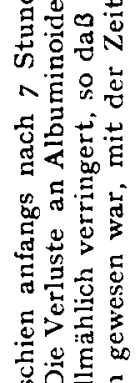 & 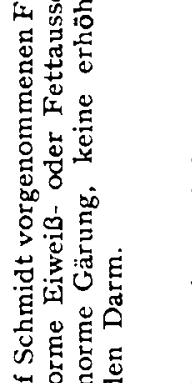 & 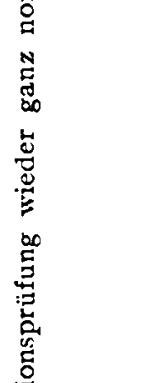 \\
\hline 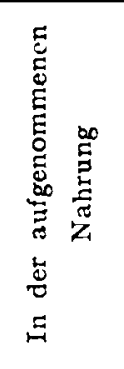 & 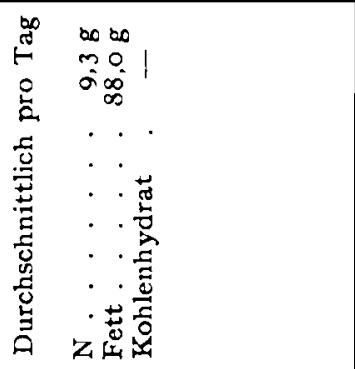 & 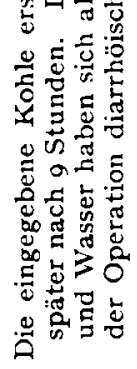 & 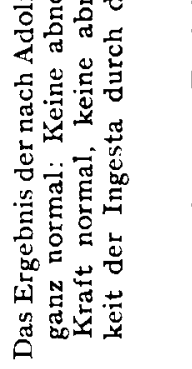 & 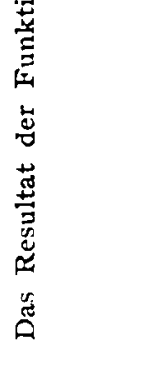 \\
\hline 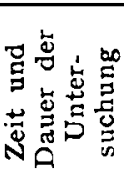 & 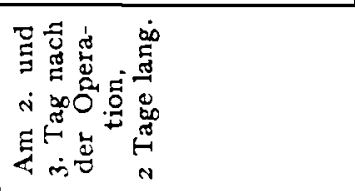 & 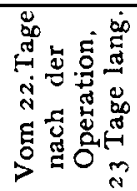 & 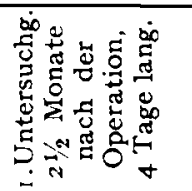 & 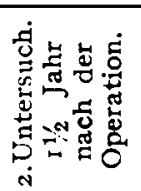 \\
\hline 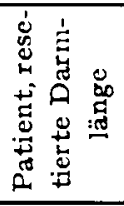 & 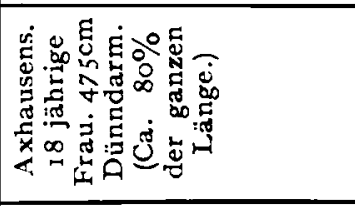 & 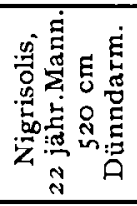 & 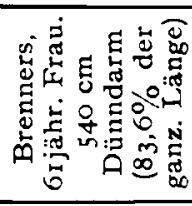 & \\
\hline 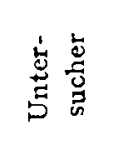 & 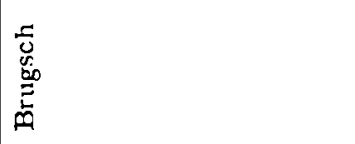 & $\stackrel{\vec{g}}{\overrightarrow{5}}$ & 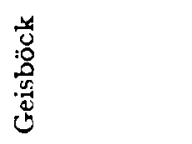 & \\
\hline 导它 & 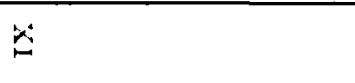 & $x$ & $\vec{x}$ & \\
\hline
\end{tabular}




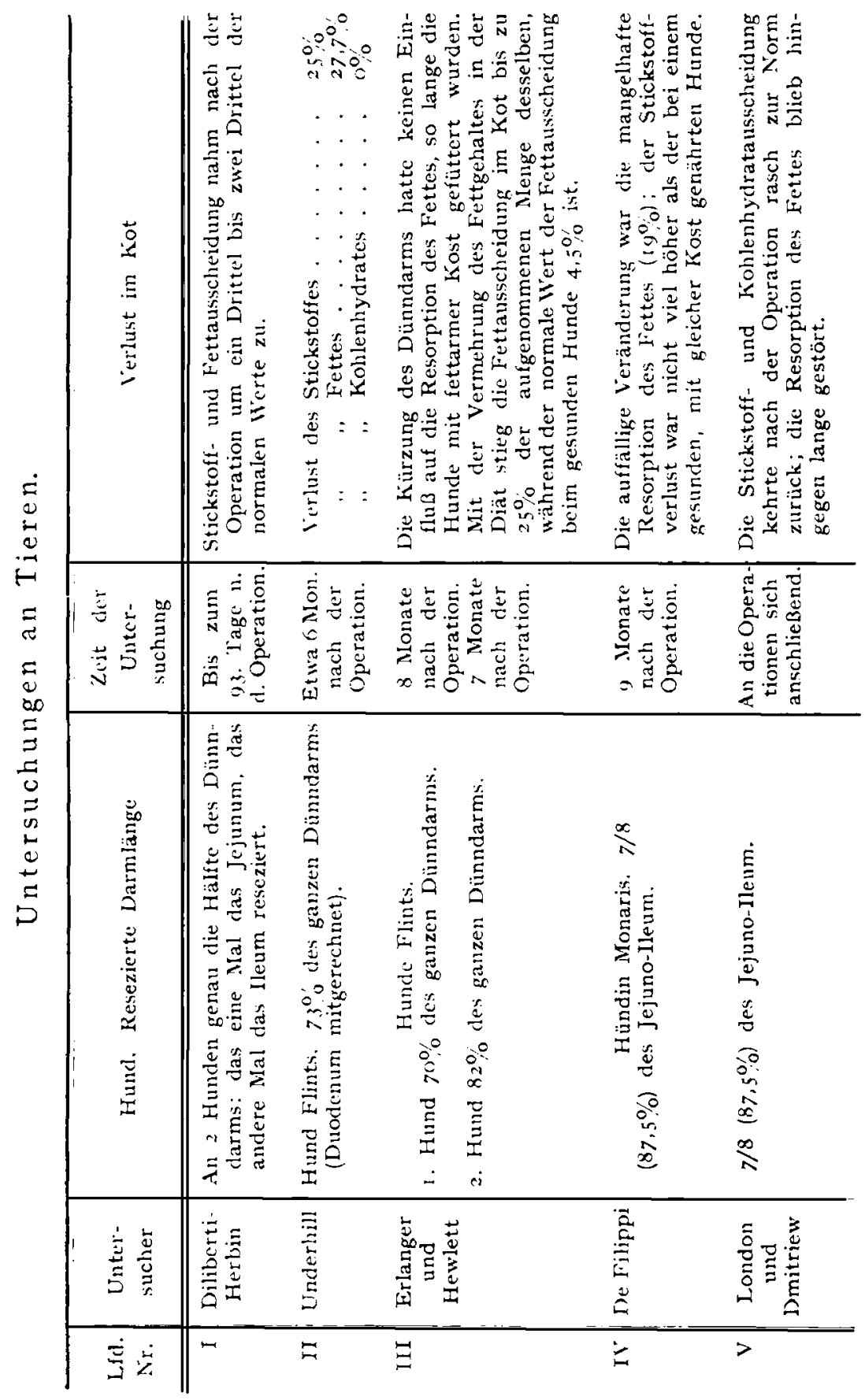


proportionell. Im letzten Jahre habe ich die Länge des Mesenterialdarms von einigen Lebenden bei Gelegenheit der Laparo. tomien nach Sappey gemessen. Das Resultat ist wie folgt:

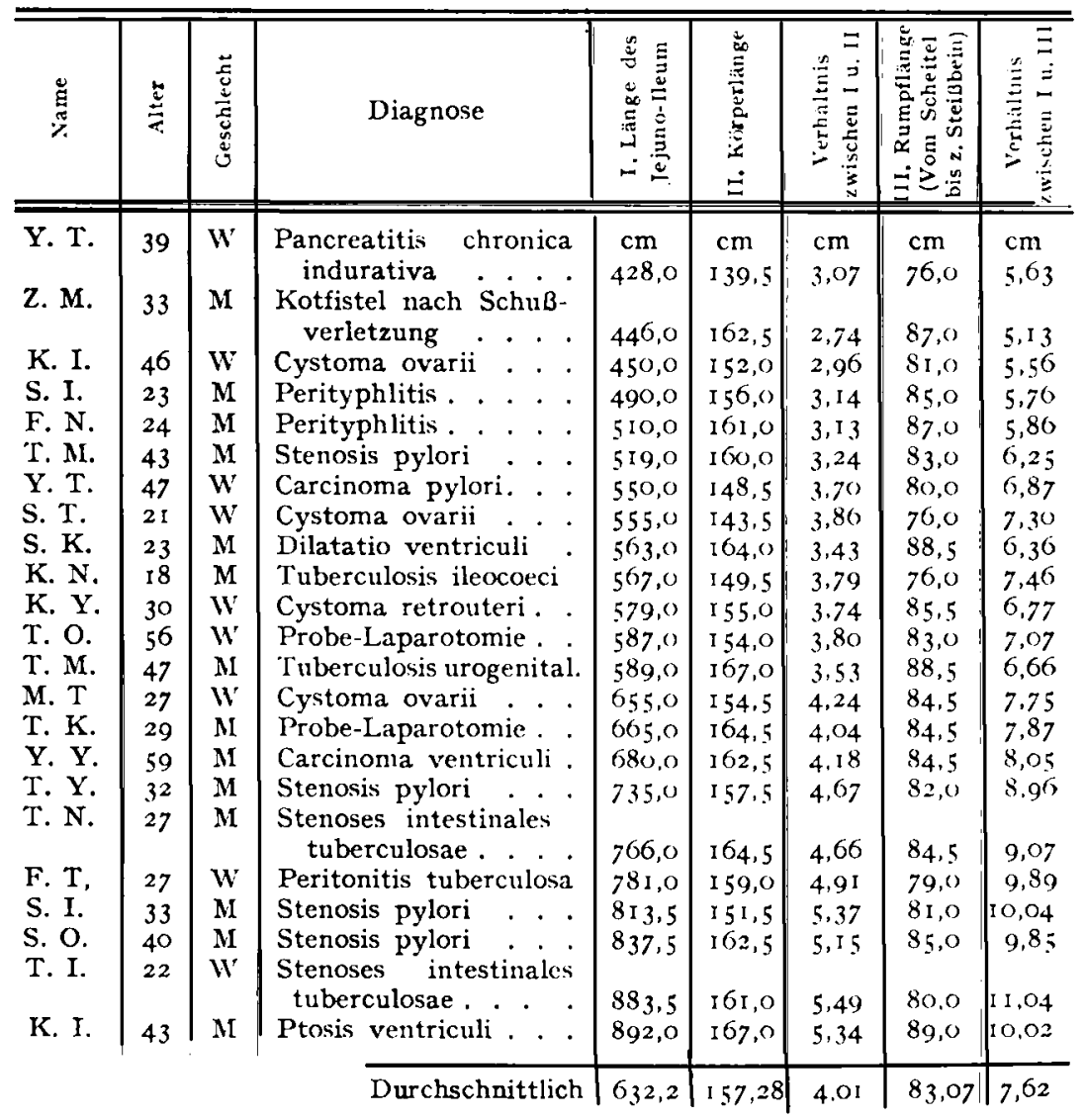

Die Länge des Dünndarms schwankt bei meinen obigen Fällen zwischen $428 \mathrm{~cm}$ und $892 \mathrm{~cm}$, indem der längste Dünndarm über doppelt so lang ist wie der kürzeste. Das Verhältnis zwischen Körper- und Darmlänge schwankt zwischen 2,74 und 5,49 $\mathrm{m}$ und dasselbe zwischen Rumpf- und Darmlänge zwischen 5, 13 und II,04 m. Die Darmlänge ist in der Tat von verschiedenen Momenten abhängig, wie Körper- bzw. Rumpflänge, Menschenrassen, Geschlecht, Alter, Lebensweise, besonders Art der Nahrung, Erkrankungen des Darms usw. So zeigt die aus der 
Körper- bzw, Rumpflänge gerechnete Darmlänge, wie Beneke tut, keinen richtigen Wert. Bei der Resektion könnte man somit an der absoluten Länge des resezierten Darms nicht so gut die Schwere der Operation erkennen wie an der relativen Länge desselben selbst. Es wäre sehr wünschenswert, daß der Operateur zunächst die ganze Darmlänge messe, dann eventuell den krankhaften Teil entferne.

Endlich soll die Lokalisation der Erkrankungen am Darme nicht unberücksichtigt bleiben.

Schon anatomisch unterscheidet sich das Jejunum vom Ileum sehr deutlich. Nach R a uber habe das erstere weiteres Lumen. dickere Wandung, reichlichere Gefäße, mehr Falten und zahlreichere Zotten als das letztere. Auch physiologisch scheinen Unterschiede vorhanden $\mathrm{zu}$ sein. Nach $\mathrm{N}$ a ga no sollen Lösungen von Rohr. zucker, Maltose und Milchzucker beim Hunde vom Jejunum besser resorbiert werden als rom Ileum. Nach Diliberti-Herbin sollen dic Störungen in Fettresorption nach Resektion des Leerdarms länger bestehen als nach der des hrummdarms. Auch Trze. bicky sagt: "dal unter sonst gleichen Verhältnissen in der Mehrzahl der Falle die Folgen einer Darmresektion am Anfangsteile des Jejunum schwerer ins Gewicht zu fallen pflegen, als jene einer Resek. tion weiter gegen die Ileocöcalklappe zu." L i e b l e in fand hingegen. daß der untere Teil des Dünndarms sowohl Peptonlösung wie Fett mehr resorbiere als der obere. T ak a y a u konnte bei seinen Hunden keinen Unterschied bemerken bezüglich der Folgen je nach der Ausschaltung des Jejunum oder Ileum.

Was nun die Ergebnisse der Verdauungs - und Resorptionsprüfungen nach Darmresektion anbetrifft, so bleibt noch viel $z u$ wünschen übrig. Die bis jetzt angestellten Untersuchungen sind nicht einheitlich in bezug auf den Zeitverlauf nach der Operation und auf die Menge der gegebenen Nährstoffe.

Bald wurden sie unternommen gleich nach der Operation, wie von Brugsch beim Fall $A x h$ a usens, bald erst io Jahre darnach, wie von $Z$ usch beim Fall Barths. Bald wurde gegeben täglich $14,9 \mathrm{~g}$ Stickstoff und $37,4 \mathrm{~g}$ Fett, wie von Onodera und I a no beim Fall $M$ i y akes, bald täglich ca. $32 \mathrm{~g}$ Stickstoff und $100 \mathrm{~g}$ Fett wie von $\mathrm{Pla}$ u t beim Fall Schlatters. Kein Wunder, daB man manchmal bei den Patienten mit einer sehr ausgedehnten Darmresektion ein besseres Verdauungs- und Resorptionsvermögen findet als bei solchen mit einer weniger ausgedehnten. Payr sagt mit Recht: „Da es offenbar längerer Zeit bedarf, um die Anpassung des 
reduzierten Darmkanals zur Ausbildung kommen zu lassen, dürften Stoffwechselversuche - längere Zeit nach der Operation unternommen - etwas andere Werte ergeben als kurz nachher angestellte."

Es versteht sich von selbst, daß auch die $Q$ ualität und $\mathrm{Q}$ uantität der gegebenen Nahrung dabei von großer Bedeutung ist. Führt man z. B. eine übermäßige Menge Speise zu, so hat selbst eine gesunde Person einen erheblichen Verlust im Kot zur Folge, während ein mehr oder weniger beschädigter Darm die Zufuhr von einer mäßigen Menge ziemlich gut verträgt.

Es ist eine auffällige Tatsache, daß eine Anzahl von Kranken nach der Operation ihren Emährungszustand unverändert beibehielten oder sogar etwas dicker wurden trotz des Vorhandenseins von Störungen in bezug auf die Verdauung und Resorption der Nahrung, besonders des Stickstoffes und des Fettes.

Um es erklären zu können, nehmen die einen Autoren in der kompensatorischen Hypertrophie und Hyperplasie des übrig bleibenden Verdauungskanals ihre Zuflucht, während die anderen auf dic reichlichere Zufuhr der geeigneten Nahrung Gewicht legen.

Was zunächst die letztere Annahme angeht, so hatte Barker Gelegenheit, 3 Patienten nach einigen Jahren wieder zu laparotomieren und fand stets die Darmwand verdünnt, mit träger Peristaltik. Auch D e $\mathrm{nk}$ konstatierte bei Sektion der berühmten Patientin Brenners mit Resektion eines $540 \mathrm{~cm}$ langen Stückes vom Dünndarm, daß die übrig bleibenden Därme nur spärliche Windungen zeigten und auffallend dünn waren. Nach $\mathrm{Kukula}$ können die Möglichkeit einer schnelleren und ausgiebigeren Resorption von seiten des zurückgelassenen Dünndarms, das experimentell nachgewiesene Substitutionsvermögen des Dickdarms, endlich der Ersatz durch häufigere und gewählte Nahrungszufuhr als Kompensationsvorrichtungen nach ausgedehnten Dünndarmverkürzungen im menschlichen Organismus fungieren.

Schlat te r sagt: ,, Meine Beobachtungen stützen auch nicht die Ansichten L a uwers u. a., daB gewöhnlich auch beim Menschen eine Hypertrophie der zurückgebliebenen Darmwand die Darmverkürzung allmählich kompensiere. . . . . Die Kompensation liegt wohl in erster Linie, wie oben hervorgehoben, in der Nahrungszufuhr. Je größer die Menge und je leichter die Assimilation der eingeführten Stoffe ist, um so unauffälliger zeigen sich die Störungen." $\mathrm{R}$ i v a ist derselben Ansicht, indem der von ihm untersuchte Patient $F$ a n t in os 
dic mangelhafte Ausnutzung der Nahrung durch eine vermehrte Aufnahme derselben zu kompensieren schien.

Auch $\mathrm{T}$ a k a y a s meint, daß die Erhaltung des Körpergewichts bzw. Wiederherstellung des Ernährungszustandes nach der Operation wesentlich ron der Beschaffenheit und Menge der zugeführten Nahrung abhängig sei.

Im Gegensatz zu den eben angeführten Angaben glauben einige Autoren experimentell eine wahre Hypertrophie und Hyperplasie der Darmwand bewiesen zu haben.

So fand Sen $n$ bei seinen Hunden stark verdickte Schichten mit ungewöhnlichem Gefäßreichtum, Dilatation und Verlängerung des bleibenden Darmabschnittes; freilich bemerkt er: „, but as a rule the increased functional activity is not adequate to make up for the great anatomical loss."

If n a ri sah bei den Hunden, die größere Resektionen überlebten, eine Hypertrophie und Hyperplasie der Schleimhaut, welche fast zweimal so dick war wie bei normalen Tieren. Bei den Tieren hingegen, die die Operation nicht überstanden, war die Schleimhaut dünn, mit niedrigen Zotten.

Bei einigen Hunden $\mathrm{N}$ ag a nos war die Darmwand sehr verdickt und derb anzufühlen; das Volum des Dünndarms war so stark vergrößert, als wenn man einen Dickdarm vor sich hätte. Mikroskopisch zeigte sich einc sehr ausgeprägte Hypertrophie der Darmzotten, sowie der Muscularis.

Flint sah an Hunden nach Resektion von $50 \rightarrow 70$ Proz. Dünndarm eine enorme Hypertrophie und Hyperplasie der Zotten.

Evans und Brenizer konstaticrten makroskopisch bei Resektion von 86,5 Proz. des ganzen Darms nach 30 Tagen eine deutliche Hyperplasie. bei Entfernung von 87.3 Proz. nach 51 Tagen fast keine Hypertrophie und bei Abtragung von 92 Proz. nach 97 Tagen gar keine Verdickung des zurückgelassenen Darms.

Trzebicky fand bei den Tieren, welche unter den Symptomen einer hochgradigen Inanition verenden, eine hochgradige Dilatation des Darmtraktus namentlich oberhalb der Naht; nach ihm entstehe aber keine kompensatorische Hypertrophie der Darmwand, wie sie von Sien $n$ beschrieben ist.

Wie wir oben gesehen haben, sind die Ansichten der Autoren über die Grenzen der Zulässigkeit ausgedehnter Darmresektionen noch sehr geteilt. Um die Frage lösen zu können, werde ich zunächst die Krankengeschichten der bisher erfolgreich ausgeführten Darmresektionen über $2 \mathrm{~m}$ kurz referieren und nachher in meine diesbezüglichen Experimente übergehen. 


\section{Krankengeschichten.}

Fall I. Operateur Lexer. 4rjähriger Patient. Seit mehreren Wochen traten häufige kolikartige Leibschmerzen auf. In der Mitte des Hypogastrium konstatierte man eine kindskopfgroße Geschwulst. Beim Eröffnen der Bauchhöhle kam eine Mesenterialgeschwulst zum Vorschein. Der Tumor wurde samt der Darmschlinge exstirpiert, welche mit der Vorderfläche desselben verwachsen war. Laterale Enteroanastomose. Ungestörter Verlauf. Schon 6 Wochen nach der Operation vertrug der Kranke gewöhnliche Krankenkost. I $1 / 2$ Jahr nach der Operation fühlte er sich vollkommen gesund und konnte alles nach Belieben essen, ohne Beschwerden dadurch zu bekommen. Stühle täglich zweimal, von normaler Farbe und Konsistenz. Histologisch war die Geschwulst ein Fibrom. Das resezierte Darmstück war am gehärteten Präparate ca. $2 \mathrm{~m}$ lang.

Fall 2. Operateur Petersen (zitiert nach Ruschhaupt). Ein 3ojähriger, kräftiger Mann wurde wegen einer Stichverletzung des Unterleibs aufgenommen. Die Wunde lag unterhalb des Nabels. Der außerhalb der Bauchhöhle befindliche, eingeklemmte Darmabschnitt wurde abgetragen und die beiden Darmenden durch eine zirkuläre Naht miteinander vereinigt. Das resezierte Darmstück war Ileum und betrug $202 \mathrm{~cm}$ in der Länge. Der Kranke erholte sich in äußerst kurzer Zeit ohne irgend eine Verdauungsstörung.

Fall 3. Operateur Koeberl é. 22jährige Patientin. Seit 2 bis 3 Jahren kolikartige Anfälle, welche im letzten Jahre häufiger und intensiver wurden. Bei der Laparotomie wurden 4 starke Darmstrikturen konstatiert. Man exzidierte ein $205 \mathrm{~cm}$ langes Darmstück und befestigte beide ligierte Stümpfe an den unteren Winkel der Bauchwunde. Erst am 3. Tage führte man Enteroanastomose aus. Heilungsverlauf nicht gestört. Die Operierte wurde vom 12. Tage an mit fester Nahrung genährt. Nach 6 Wochen schloB sich die Bauchwunde narbig und die Kranke genas ohne Verdauungsstörungen.

Fall 4. Operateur Enderlen (zitiert nach Lauenstein). 58 jähriger, magerer Mann. Seit 8 Jahren doppelseitige Leistenbrüche. Herniotomie ca. I2 Stunden nach der Einklemmung der linksseitigen Hernie. Im Bruchsack lagen zwei Dünndarmschlingen nebeneinander. Durch einen leichten Zug an den inneren Schenkeln der Außenschlingen kam die Innenschlinge aus der Bauchhöhle heraus. Die 3 Schlingen, im ganzen $207 \mathrm{~cm}$ lang, wurden reseziert, mitsamt einem großen Teil des zugehörigen Mesenterium. Seit-zu-Seit-Anastomose, Radikaloperation nach $\mathrm{B}$ a s sini. In den ersten 2 Tagen blutige Stühle, nachher ganz glatte Rekonvaleszenz.

Fall 5. Operateur Kocher (zitiert nach Trzebicky). Bei einem Manne mit Dünndarmzerreißung infolge eines Eisenbahnunglücks wurde ein $208 \mathrm{~cm}$ langes Darmstück reseziert. Heilung 
per primam. 6 Monate später klagte Patient nur noch über leicht auftretende Durchfälle, falls er mit dem Essen nicht sehr vorsichtig war.

Fall 6. Operateur Dreesmann. 37jähriges Mädchen. Am letzten Abend klemmte sich die rechtsseitige Schenkelhernie ein. Herniolaparotomie. Die im Bruchsack befindlichen Darmschlingen um $180^{\circ}$ gedreht und in dieser Lage durch einen Netzstrang fixiert. Die ganze gangränöse Partie des Darms, $215 \mathrm{~cm}$ lang, reseziert. Das anale Darmende an und für sich geschlossen und das orale seitlich ins Colon ascendens eingepflanzt. Nach 5 Wochen völlig geheilt entlassen. 7 Monate später teilte die Kranke mit, daß sie trotz der vorsichtigen Diät täglich zweimal breiige Stühle hatte, wenn auch Körpergewicht dadurch nicht besonders vermindert wurde.

Fall 7. Operateur Karlow. 33jähriger Mann. Darmgangrän infolge einer zweifachen Strangulation. Resektion von einem $215 \mathrm{~cm}$ langen Stück Dünndarm und zirkuläre Darmnaht. Der Kranke wurde vollständig wieder hergestcllt.

Fall 8. Operateur Axhausen. Ein 4rjähriger Mann wurde wegen Erscheinungen von Pylorusstenose operiert. Anlegung einer Gastroenterostomie. Zunächst glatter Verlauf. Am I I. Tage post operationem Einsetzen ron Symptomen der Darmstenose. 5 Tage darnach Relaparotomie. Man fand die Anastomosenstelle ganz in Ordnung, nur war der abführende Darmschenkel auffallend stark gefüllt. Beim Herausholen des nächsten Dünndarmteils fand man handbreit unter der Anastomose eine Dünndarmstriktur, offenbar tuberkulöser Natur, worauf abwärts noch etwa 12 weitere Strikturen folgten. Resektion des veränderten, $215 \mathrm{~cm}$ langen Darmabschnittes, mit Fortnahme der geschwollenen Mesenterialdrüsen. Glatter Verlauf. Der Kranke stcllt sich 7 Monate post operationem wieder in der Klinik ein und zeigte cine Gewichtszunahme um 40 Pfd.!

Fall 9. Operateur II ikulicz (ziticrt nach Rothe). 3ojähriger Mann. Vor 3 Tagen klemmte sich die linksseitige äußere Leistenhernie ein. Bei der Herniotomie fand man das im Bruchsack befindliche große Darmkonvolut an vielen Stellen gangränös verändert. $215 \mathrm{~cm}$ lange Darmresektion und seitliche Enteroanastomose. Körpergewicht zugenommen, geheilt entlassen. Im nächsten Jahre teilte Patient mit, daß er absolut diätlos lcbte, einen regelmäßigen Stuhlgang hatte und viel arbeitsfähiger war als vor der Operation, da der Bruch nic wieder sich gezeigt hatte.

Fall 10. Operateur Dekonski (zitiert nach I st o min). $220 \mathrm{~cm}$ lange Dünndarmresektion mit Erfolg.

Fall iı. Operateur Thon. Ein 64jähriger Mann erkrankte plötzlich an lleus und wurde $2+$ Stunden nach dem ersten Eintritt des Leidens operiert. Beim Eröffnen des Peritoneum zeigte sich ein unentwirrbares Dünndarmkonvolut; es wurde ein $220 \mathrm{~cm}$ langes Stück 
Dünndarm entfernt. Um die Operation schnell zu beenden, wurden beide Darmenden in die Bauchwunde eingenäht. Der Patient genas.

Fall 12. Operateur Kouwer (zitiert nach Flint). Eingeklemmte Hernie. Resektion des gangränösen Darms $22+\mathrm{cm}$ in der Länge. Heilung. Keine Verdauungsstörung.

Fall I3. Operateur Sepherd. 28jähriger Mann. Seit einem Jahre bemerkte er einen allmählich wachsenden Tumor in der Bauchhöhle. Nie Störungen der Darmpassage. Bei der Operation fand man eine übermannskopfgroße Mesenterialgeschwulst, mit lleum und Colon transversum verwachsen. Die Geschwulst wurde exstirpiert, samt einem $234 \mathrm{~cm}$ langen Stück Ileum. Enteroanastomose. Der Kranke erholte sich rapid und verlieB 5 Wochen post operationem das Spital. Einen Monat später schrieb er, daß er nach Entlassung sehr häufig an Durchfällen und zeitweise an Kolikschmerzen litt. Sein Körpergewicht nahm in der kurzen Zeit um etwa 25 Pfd. zu. Ca. 8 Monate darnach befand sich Patient vollkommen gesund, Stuhlgang wurde ganz normal.

Fall I4. Operateur $\mathrm{Ku} \mathrm{k} \mathrm{u} \mathrm{la.} 38$ jährige Arbeiterfrau. Vor 6 Jahren litt sie an Pleuritis duplex. Seit Halbjahr stellten sich häufig Bauchschmerzen und weiche Stühle ein. Bei der Laparotomie fand man einen ziemlich scharf begrenzten Mesenterialtumor mit einigen regionären Drüsenanschwellungen. Der Tumor wurde samt dem damit fest verwachsenen, $237 \mathrm{~cm}$ langen Stück Ileum entfernt. End-zu-EndAnastomose. 3 Monate nach der Operation geheilt entlassen. Volle 2 Jahre hindurch fühlte sich die Kranke sehr wohl, befreit von Darmstörungen. Später stellten sich leichte Darmkoliken wieder ein und eine diffuse Resistenz entstand im Abdomen rechts von der Wirbelsäule. Einige Monate darnach traten hohes Fieber, Schmerzhaftigkeit an der Ileozökalgegend, Durchfälle usw. ein. Eiter wurde durch Incisionen entleert. Sie starb schließlich 2 Jahre und 9 Monate nach der ersten Operation. Bei der Sektion wurde konstatiert, da $B$ der zurückgebliebene Dünndarm I Io cm lang war. Der exstirpierte Tumor war von tuberkulöser Natur.

Fall 15. Operateur Miyake. 57jähriger Bauer. Bauchtumor mit Stenosenerscheinungen des Darms. Stuhlgang täglich einmal. Nach Eröffnung der Bauchhöhle fand man ein derbes, unregelmäßig gestaltetes, mannsfaustgroßes Konglomerat des Cöcum und einen Teil von Ileum und Colon ascendens. Ferner saßen 8 tuberkulöse Geschwüre am unteren Teile des lleum zerstreut. Die tuberkulös affizierte Darmpartie samt den intumeszierten regionären Drüsen abgetragen und die seitliche Anastomose zwischen Ileum und Colon transversum angelegt. Das resezierte Darmstück bestand aus einem 225,5 cm langen Dünndarm- und $13 \mathrm{~cm}$ langen Dickdarmteil. Glatter Verlauf. 36 Tage post operationem geheilt entlassen. 7 Monate nach der Operation konstatierte man eine Körpergewichtszunahme 
um $9,5 \mathrm{~kg}$ und nach I Jahre das rollkommene Wohlbefinden des Patienten.

Fall r6. Operateur $\mathrm{H}$ a rris. 33jähriger Mann. Seit 5 Jahren stellten sich zeitweise heftige Bauchschmerzen ein, zuletzt traten Ileussymptome auf. Bei der sofort ausgeführten Laparotomie kam eine gangränöse Darmschlinge zum Vorschein, welche von einem Mesenterialstrang eingeschnürt und an der hinteren Bauchwand fixiert war. Der Strang wurde durchschnitten und das nekrotisierte $239 \mathrm{~cm}$ lange Stück Dünndarm reseziert. Beide Darmenden, Cöcum und Ileum mittels eines Murphyknopfes seitlich anastomosiert. 3 Wochen nach det Operation wurden ihm alle Speisearten gestattet; er wog dann schwerer als bei der Aufnahme und verließ das Hospital als geheilt. Er fühlte sich seither stets wohl, neigte nur zu Durchfällen und vermied einen übermäßigen Genuß von grünen Gemüsen.

Fall 17. Operateur B renner (zitiert nach Denk). Erfolgreiche Resektion ron einem $250 \mathrm{~cm}$ langen Dünndarmstück wegen Intussuszeption, verursacht durch einen Darmpolypen. Später keine Verdauungsstörungen.

Fall 18. Operateur Enochin (zitiert nach Istomin). Darmresektion iiber $250 \mathrm{~cm}$. Heilung.

Fall 19. Operateur $\mathrm{H}$ ayes (zitiert nach Blayney). Ein rojähriger Knabe wurde von einem schwer beladenen Wagen über die Lendengegend überfahren. Darauf wiederholtes Erbrechen und heftige Bauchschmerzen aufgetreten. Als man am nächsten Morgen die Bauchhöhle aufmachte, fand man einen quer verlaufenden Mcsenterialriß, 3 Zoll rom Darmansatz entfermt. Das zugehörige lleum war nekrotisch, wenn auch noch nirgends perforiert und wurde in der Länge von $255 \mathrm{~cm}$ reseziert. Vom 12. Tage an traten Durchfälle cin, die etwa 7 Wochen andauerten. Bei der Entlassung, 3 Monate nach der Operation, war der Appetit ungemein stark. Zu Hause häufig Erbrechen, Bauchschmerzen, Durchfälle, starke Abmagerung. Bei der letzten Untersuchung, ca. 1, Jahr nach der Entlassung, fand man ihn sehr gebessert: Körpergewichtszunahme, Appetit sehr groß3. Stühle meist zwcimal täglich, zuweilen sehr lästig.

Fall 20. Operateur Peck (zitiert nach $\mathrm{H}$ arris). Bei einer Frau wurde ein $258 \mathrm{~cm}$ langes Darmstück reseziert, wegen des Fntschlüpfens desselben durch den rupturierten Literus ins Becken. Heilung.

Fall 21. Operateur L a uwers. Eine 65jährige Frau bekam eine Darmfistel infolge einer eingeklemmten Bauchwandhernie. Beim Versuche, die miteinander verwachsenen Darmschlingen zu lösen, rissen sie an mehreren Stellen ein. So wurde der Dünndarm in der Länge von $265 \mathrm{~cm}$ mit Erfolg reseziert.

Fall 22. Operateur Park. 24jähriger Mann. Seit 5 Tagen Fieber, heftige Bauchschmerzen, Erbrechen und Stuhlverstopfung. 
Der Bauch war ein wenig aufgebläht und fühlte sich etwas rigid an. Bei der Laparotomie fand man eine Verwachsung des großen Netzes mit den Darmschlingen und eine Menge seropurulenter Flüssigkeit zwischen denselben. Cöcum und lleum waren an mehreren Stellen im Begriffe zu perforieren. Der primäre Krankheitsherd schien die gangränöse Appendicitis zu sein. So wurde das Colon ascendens mit einer Darmschlinge, dicht oberhalb der erkrankten Partie, seitlich anastomosiert und das dazwischen liegende, $265 \mathrm{~cm}$ lange Stück Ileum nebst einem großen Teil des Blinddarms reseziert. Patient bekam einen enorm großen Appetit und erholte sich bald, so daß sein Körpergewicht II Monate später größer wurde wie vor der Operation.

Fall 23. Operateur Payr. 39jährige Frau. Seit mehreren Jahren hatte sie die rechtsseitige Leistenhernie. Vor 3 Tagen bekam sic Bauchschmerzen und nach Reposition der Hernie raten ausgeprägte Erscheinungen innerer Inkarzeration ein. Bei der Laparotomie konstatierte man eine große Partie des Dünndarms schwärzlich verfärbt, infolge einer Strangulation. Resektion von einem $265 \mathrm{~cm}$ langen Stück Darm, oralwärts von der Stelle $40 \mathrm{~cm}$ oberhalb der Ileozökalklappe. Termino-Terminal-Anastomose. Am 3. Tage entleerte Patientin einen normalen Stuhl. I Monat später erhielt sie Mohnkörner zu essen, welche 31 Stunden darnach im Stuhle erschienen. Einmal bekam sie versuchsweise nur Fleisch, andermal nur Kohlenhydrat; der Stuhl enthielt sowohl makro- wie mikroskopisch nichts abnormes. Am Ende des 2. Monats völlig geheilt entlassen. Nach 5 Monaten fühlte sie sich ganz gesund. Sie hatte einen guten Appetit und empfand öfters Hunger. Stühle waren weich und nicht diarrhöisch, unverdaute Speisereste gingen nie ab. 3/4 Jahr nach der Operation teilte sie mit, daB sie sich immer noch sehr wohl befand.

Fall 24. Operateur Pokotilo. Resektion von einem $270 \mathrm{~cm}$ langen Stück Dünndarm wegen der eingeklemmten, rechtsseitigen Leistenhernie. Patient genas und fühlte sich nachher sehr wohl.

Fall 25. Operateur Kopfstein. Eine 35jährige heruntergekommene Frau wurde operjert wegen typischer Anfälle, welche auf Stenosen des Darms hinwiesen. Bei der Laparotomie fand man 6 zirkuläre, ziemlich durchgängige tuberkulöse Strikturen an verschiedenen Stellen des Dünndarms in ungleicher Entfernung voneinander. Die Gesamtlänge des erkrankten Darms betrug $275 \mathrm{~cm}$. Resektion der kranken Partie, zirkuläre Naht. Vom Dünndarm blieb ein etwa $172 \mathrm{~cm}$ langes Stück übrig. In den ersten Tagen hatte die Kranke Durchfälle, welche schon am 6. Tage aufhörten. Rapide Zunahme des Körpergewichtes. Vor der Operation wog sie $42,7 \mathrm{~kg}, 1 / 2 \mathrm{Jahr}$ später $54 \mathrm{~kg}$. Sie nahm zu Hause gewöhnliche Kost zu sich und konnte alle schweren landwirtschaftlichen Arbeiten verrichten; der Stuhl war immer regelmäBig. I Jahr nach der Entlassung gebar sie 
ein normal entwickeltes Kind, und als sie $1 / 2$ Jahr später wieder schwanger wurde, starb sie angeblich an einem septischen PuerperalprozeB.

Fall 26. Operateur Maydl (zitiert nach Kukula). Eine 33jährige stark erschöpfte Frau bekam seit 10 Monaten Stenosenerscheinungen des Darms. Es wurde eine harte hühnereigroße höckerige Geschwulst an der Ileozökalgegend konstatiert. Bei der Operation wurde ein infolge von Unterbindung der Gefäße dunkelblau verfärbtes, $284 \mathrm{~cm}$ langes Stück Ileum nebst Colon ascendens reseziert. Unterdessen kollabierte die Kranke hochgradig; um die Operation schnell zu beenden, wurde ein Anus praeternaturalis gebildet. Vom Anfang der 3. Woche an trat eine stets zunehmende Verschlechterung des Ernährungszustandes ein. Appetit erlosch vollkommen, und endlich 23 Tage nach der Operation erlag sie trotz der künstlichen Ernährung einer Inanition. Bei der Sektion fand man eine vorgeschrittene Lungenphthise und umschriebene Abszesse zwischen Darmschlingen. Der exstirpierte Tumor war histologisch von tuberkulöser Natur.

Fall 27. Operateur $\mathrm{Childe.} \mathrm{59jährige} \mathrm{gut} \mathrm{genährte} \mathrm{Frau.}$ Vor 17 Jahren wurde sie einmal wegen eingeklemmter rechtsseitigen Schenkelhernie operiert. Abermalige Einklemmung derselben. Bei der Herniotomie fand man die im Bruchsack befindlichen Darmschlingen schwärzlich verfärbt. Die Gangrän erstreckte sich noch weit hinauf bis an die Schlingen in der Bauchhöhle. Resektion des unteren Abschnittes vom Dünndarm $289 \mathrm{~cm}$ lang, seitliche Enteroanastomose. Anfangs war der Verlauf ein befriedigender: Appetit gut, Stuhlgang einmal täglich, teilweise geformt. Später trat Diarrhöe und Körpergewichtsabnahme ein. Sie war mit ihrer Diät sehr vorsichtig und nahm eine kleine Dose Opium ein. 7 Monate nach der Operation ging sie erschöpft zugrunde.

Fall 28. Operateur Lor enz. 55jähriger Beamter mit einem 'Tumor in der Ileozökalgegend. Die ganze Tumormasse samt dem verwachsenen Ileum und Colon ascendens bis zur Flexura hepatica in toto exstirpiert. Die Länge des resezierten Darms betrug im kollabierten Zustande $292 \mathrm{~cm}$. Beide Enden, Dick- und Dünndarm termino-lateral anastomosiert. In den ersten Tagen traten profuse wässerige Stuhlentleerungen ein, welche nach einigen Tagen seltener und konsistenter wurden. Appetit gut, Erholung rasch. Etwa 6 Wochen nach der Operation befand sich der Kranke wohl und nahm an Körpergewicht zu. Stuhlgang bloß zweimal täglich und zwar dünn- bis dickbreig.

Fall 29. Operateur Göb e 11. Bei einem 30 Jahre alten Manne traten plötzlich Erscheinungen innerer Einklemmung ein, und es wurde 28 Stunden nach dem Einsetzen der Symptome operiert. Man fand eine große Partie der Dünndarmschlingen schwärzlich verfärbt; 
es war sowohl eine wahre Knotenbildung, wie eine Achsendrehung gebildet. Das veränderte Stück vom Ileum in der Länge von $297 \mathrm{~cm}$ reseziert und eine seitliche Ileokolostomie angelegt. Nach 6 Wochen völlig geheilt entlassen. Patient befand sich $10 \frac{1}{2}$ Monate nach der Operation sehr wohl. Er wog schwerer als früher und war vollkommen arbeitsfähig als Werftarbeiter. Niemals Diarrhöe, obwohl er zu seiner gewöhnlichen Kost zurückkehrte; er verspürte nie Heißhunger und nahm in der Regel nicht viel Nahrung zu sich. Der Stuhl war meist weich, geformt, täglich ein- bis zweimal, sehr selten dreimal und von normaler brauner Farbe. Nur bei sehr fettreicher Nahrung wurde alles Fett nicht völlig resorbiert. 6 Jahre nach der Operation immer noch vollkommen erwerbsfähig.

Fall 30. Operateur Fantino. Eine 4rjährige Frau fühlte seit 4 Jahren leichte Bauchschmerzen an der rechten Seite. Vor I Jahre wurde bei ihr ein Bauchtumor konstatiert, der in den letzten Monaten rapid zu wachsen anfing. Bei der Laparotomie fand man ein großes Fibromyoma uteri, stark vaskularisiert und zwischen die Mesenterialblätter hineingewachsen, so daß der Dünndarm die Geschwulst kronenartig umgab. Die Geschwulst samt dem Darm und Uterus exstirpiert. Beide Darmenden an und für sich geschlossen und eine seitliche Anastomose zwischen dem Anfangsteil des Colon ascendens und dem Dünndarm hergestellt. Das resezierte. Darmstück gehörte dem unteren Dünndarmabschnitt und betrug ca. $3 \mathrm{~m}$ in der Länge. Vom 6. Tage an traten unstillbare Durchfälle ein, im Anschluß an eine ziemlich reichliche Nahrungsaufnahme. Alle möglichen internen Mittel und Diät blieben wirkungslos. Stühle waren stets flüssig, gallenreich; Milch, Eier und Fleisch wurden unverdaut entleert. Etwa I Monat post operationem ging Patientin erschöpft zugrunde.

Fall 31. Operateur Fantin o. 6ojähriger Bauer. Einklemmung einer rechtsseitigen kindskopfgroßen Leistenhernie. Herniotomie. Resektion des unteren Teils Ileum, $310 \mathrm{~cm}$ in der Länge. Seitliche Enteroanastomose. Heilung per primam. Trotz des häufigen Stuhlganges war keine Abmagerung bemerkbar. I Jahr später befand sich Patient in der besten Gesundheit und wurde sogar dicker wie vor der Operation.

Fall 32. Operateur MI on profit. 42jähriger Mann. Bei der Radikaloperation eines mannskopfgroßen, unaufhörlich wachsenden, unbeweglichen Inguinalbruches resezierte man das mit der Wand fest verwachsene Darmkonvolut, aus einem $230 \mathrm{~cm}$ langen Stück Ileum und einem $80 \mathrm{~cm}$ langen Stück Dickdarm bestehend, in toto und anastomosierte beide Darmenden seitlich. In den ersten Tagen traten nach dem Essen gewöhnlicher Kost Durchfälle ein, welche jedoch gänzlich stillstanden, als Patient die vegetabilische Nahrung statt der animalischen zu sich nahm. Schnelle Erholung. 4 MIonate nach der 
Operation fühlte er sich ganz wohl und klagte nur dann ïber Diarrhöe, falls er Fleisch verzehrte. 6 Jahre post operationem wurde das beste Wohlbefinden konstatiert.

Fall 33. Operateur Ghedini. 52jährige Tagelöhnerin. Inkarzeration der rechtsseitigen Inguinalhernie. Bei der am nächsten Tage ausgeführten Herniotomie wurde ein $315 \mathrm{~cm}$ langes gangränöses Stück Dünndarm reseziert. Beide Darmstümpfe mittels eines Murphyknopfes termino-terminal vereinigt. Am Io. Tage Abgang des Knopfes. I'atientin klagt immer über die allgemeine Mattigkeit. Stühle stets diarrhöisch, anfangs drei- bis viermal täglich, später vier-bis sechsmal. 25 Tage nach der Operation mit einer narbig geheilten Wunde cntlassen. $3^{1}$ : Monat post operationem Tod infolge von unstillbaren Durchfällen.

Fall 34. Operateur $\mathrm{Barth}$ (zitiert nach $\mathrm{Zusch}$ ). Eine 40jährige Frau klagte über eine Kotfistel, entstanden nach der Operation eines vereiterten Ovarialtumors. Es wurde eine totale Darmausschaltung vorgenommen. Als dies nun infolge der Sekretretention Beschwerden verursachte, wurde das ausgeschaltete Stück Dünndarm in der Länge von $316 \mathrm{~cm}$ reseziert. Die Kranke genas. Stühle reichlich und breiig, aber nicht diarrhöisch. I4 Jahre nach der totalen Ausschaltung ein gutes Allgemeinbefinden trotz der Unterernährung.

Fall 35. Operateur $F$ ischer. Resektion eines infolge von Brucheinklemmung und Mesenterialtorsion nekrotisierten, $316 \mathrm{~cm}$ langen Stückes rom Dünndarm. Der Kranke war nach einer achtwöchentlichen Rekonvaleszenz so gestärkt, daß er seiner gewohnten Arbeit nachgehen konnte.

Fall 36. Operateur $\mathrm{Z}$ eidler (zitiert nach Spassokuko$\mathrm{zkaja).} \mathrm{z2jähriger} \mathrm{Mann.} \mathrm{Einklemmung} \mathrm{der} \mathrm{großen} \mathrm{rechtsseitigen}$ Skrotalhernie. Bei der 2 Stunden später ausgeführten Operation fand man eine Torsion des Darms; es wurde ein $318 \mathrm{~cm}$ langes gangränöses Stück Ileum reseziert. Die beiden Enden des Darms an und für sich gescblossen und eine seitliche Anastomose mittels eines Murphyknopfes angelegt. I Jahr nach der Operation fühlte sich Patient ganz wohl.

Fall 37. Operateur Morton (zitiert nach Flint). Resektion eines $322 \mathrm{~cm}$ langen Stückes Dünndarm wegen eines Myxosarcoma mesenterii. Heilung.

Fall 38 . Operateur $\mathrm{R}$ uggi. Ein Sjühriger Knabe, der früher stets gesund war, erhielt einen sehr kräftigen StoB auf die Bauchwand, rechts rom Nabcl. Nach dem Trauma entwickelten sich Stenosenerscheinungen des Darms in einer allmählich zunehmenden Heftigkeit. Endlich traten Symptome des Ileus ein, und da die interne Behandlung wirkungslos blieb, entschlob sich der Kranke zur Operation, erst etwa 4 Monate nach der Verletzung. Bei der Laparotomie fand sich der Darm durch cincn Nctzstrang cingcschnürt, der ziemlich leicht los- 
zulösen war. Die Erleichterung hielt darauf nur einige Tage an. Bei der zweiten Operation, 8 Tage nach der ersten, konstatierte man den Darm an der eingeschnürten Stelle verengert; so wurde eine Enteroanastomose angelegt. I Woche darnach traten die alten Erscheinungen wieder auf und zum drittenmal, 2 Wochen nach der zweiten Operation, wurde die Bauchhöhle breit aufgemacht. Da die Darmschlingen durch eine umschriebene Peritonitis unlösbar miteinander verwachsen waren, wurde ein $330 \mathrm{~cm}$ langes Stück derselben, oralwärts von der Stelle, $15 \mathrm{~cm}$ oberhalb der Bauhinischen Klappe reseziert; darauf wurde eine zirkuläre Darmnaht angelegt. Sehr rasche Erholung. I4 Jahre post operationem immer im besten Wohlbefinden.

Fall 39. Operateur Sta ehlin. 47jähriger kräftiger Mann. Ein klemmung der rechtsseitigen Leistenhernie. 4 Stunden darnach ins Spital aufgenommen und sofort operiert. Ein $335 \mathrm{~cm}$ langes, gangränöses Stück Dünndarm abgetragen und End-zu-End anastomosiert. Nach 7 Wochen geheilt entlassen. 2 Monate nach der Entlassung war der Kranke als Feuerwehrmann dienstfähig wie vorher. Er aß und trank wie sonst und erreichte bald sein ursprüngliches Körpergewicht. 4 Jahre post operationem fühlte er sich ganz wohl und leistete eine schwere körperliche Arbeit ohne jede Ermüdung. Stühle täglich zwei- bis dreimal, breiig.

Fall 40. Operateur Nicola. Resektion eines $350 \mathrm{~cm}$ langen Stücks vom Dünndarm wegen einer eingeklemmten Nabelhernie. Heilung.

Fall 41. Operateur v. Eiselsberg (zitiert nach Franz). 65jährige Frau. Einklemmung einer linksseitigen Hernie seit 3 Tagen. Der Dünndarm mit der Sackwand flächenhaft verwachsen; Resektion desselben in der Länge von $3^{1 / 2} \mathrm{~m}$. Die Darmnaht kam dicht oralwärts von der Ileocöcalklappe zu liegen. Reaktionsloser Verlauf bis zum 8. Tage; dann Komplikation durch Erysipel. Exitus 25 Tage post operationem, infolge eines Kotabszesses im Mesenterium und Mesocolon. Die Darmnaht teilweise nicht gehalten. In der Lebzeit keine Verdauungsstörungen trotz des so erheblich verkürzten Darms.

Fall 42. Operateur Werelius. 2ojähriges Mädchen. Beim Versuche, verbrecherischer Weise zu abortieren, kam ein Stück Dünndarm aus der Vagina heraus, welcher mißhandelt wurde. Bei der Laparotomic kamen vom Mesenterium losgelöste, gangränös verfärbte Darmschlingen zum Vorschein, welche in der Länge von $365 \mathrm{~cm}$ reseziert wurden. Darmnaht mittels eines Murphyknopfes. Perforationsöffnungen des Uterus durch Nähte geschlossen. Verlauf nach der Operation günstig. Am 27. Tage geheilt entlassen. 2 Wochen später traten Durchfälle auf, welche etwa 14 Tage lang dauerten. 8 Monate nach der Operation lebte die Kranke. beschwerdefrei, das Körpergewicht nahm um $15,5 \mathrm{~kg}$ zu. I8 Monate post operationem konnte sie die gewöhnliche Speise zu sich nehmen. Stuhlgang meist 
einmai täglich, ausnahmsweise mehr. 3 Jahre nach der Operation volles Wohlbefinden, keine gastro-intestinalen Störungen.

Fall 43. Cperateur $\mathrm{M}$ itchell. Resektion von ca. I2 FuB $(360 \mathrm{~cm})$ langem Stück Dünndarm mit Erfolg.

Fall 44. Operateur Friedrich. 54jährige Frau, die eine Kotfistel nach der Operation einer eingeklemmten Schenkelhernie gehabt hatte, bekam peritonitische Symptome. Bei der Laparotomie fand man einen großen Dünndarmabschnitt, analwärts von der Fistel und über $3 \mathrm{~m}$ in der Länge, in peritonitischem Exsudate schwimmend. Es wurde eine Kommunikation zwischen Dünndarm und Colon transversum hergestellt, um den peritonitisch erkrankten Darmteil in der Länge von etwa $4 \mathrm{~m}$ einschließlich der Fistel auszuschalten. $\mathrm{I}^{1 / 2} \mathrm{Jahre}$ post operationem fühlte sich die Kranke ganz wohl. Sie erlag, I Jahr später, an krupöser Pneumonie.

Fall 45. Operateur Pa chet. Einem Patienten wurde ein $4 \mathrm{~m}$ langes Stück Dünndarm wegen einer irreponiblen mächtigen I.eistenhernie rescziert. Er befand sich während seines Aufenthalts in der Klinik für I Monat sehr wohl. Nach der Entlassung a $B$ und trank er wie gewohnt, was fulminante Diarrhöen zur Folge hatte. Hauptsächlich durch trockne Diät regelte sich der Stuhl sehr bald. Tod an zunehmender Kachexie.

Fall 46. Operateur $\mathrm{A} \times \mathrm{h}$ a usen. Bei einer $\mathrm{I} 8$ jährigen schlecht genährten Frau mit doppelseitiger Lungenphthise trat plötzlich Ileus ein. Bei der 32 Stunden nach Beginn der Erkrankung vorgenommenen Operation konstatierte man eine Achsendrehung des gesamten Dünndarms um $180^{\circ}$. Resektion desselben in der Länge von $475 \mathrm{~cm}$ und Anastomoscnbildung Seit-zu-Seit zwischen Jejunum und Cöcum. Der ubrig bleibende Dünndarm war ron der Flexura duodenojejunalis bis zur Stelle der Anastomose $125 \mathrm{~cm}$ lang. Die Darmpassage stellte sich unmittelbar nach der Operation wieder ein. Vom 3. Tage post operationem an hatte die Kranke des Morgens eine reichliche, dünnbis dickbreiige Stuhlentleerung und manchmal des Mittags eine zweite; später hatte sic zuweilen 3 Stühle im Laufe eines Tages. Der Appetit war nur in der 1 . Woche gut, späterhin dauernd schlecht. 82 Tage post operationem in ziemlich gutem Allgemeinbefinden und mit unverändertem Kürpergewicht entlassen. Nach der Entlassung machte die Lungenphthise rasche Fortschritte: reichlicher Auswurf, hektisches Fieber, Körpergewichtsabnahme usw. Exitus 6 MIonate nach der Operation.

Fall 47. Operateur St ol z. 40jähriger Mann. 20 Stunden nach dem Auftreten der ersten Symptome von Ileus wurde die Operation vorgenommen. Es handelte sich um cinen Volvulus des Dünndarms. Man resezierte den crkrankten mittleren 'Teil desselben in der Länge von $475 \mathrm{~cm}$. und vereinigte die beiden Darmenden End-zu-End mittels 
eines MIurphyknopfes. In der I. Woche traten außerordentlich häufige und profuse Durchfälle auf, seit dem am Io. Tage erfolgten Abgang des Murphyknopfs wurde jedoch das Allgemeinbefinden allmählich besser. Die Nahrung bestand hauptsächlich aus Vegetabilien. Wurde Fleisch verabreicht, so verursachte es sofort wieder stärkere Durchfälle. I Jahr post operationem nur eine leichte Ermüdung beim Spazierengehen.

Fall 48. Operateur $\mathrm{S}$ tor p. Ein $2 \mathrm{I}$ jähriger abgemagerter Mann bekam seit einigen Monaten Leibschmerzen, Appetitlosigkeit und Obstipation. Bei der Laparotomie fand man ein großes Mesenterialsarkom, welches samt den daraufsitzenden Darmschlingen entfernt wurde. Die Länge des mitresezierten Darms betrug $510 \mathrm{~cm}$. Die Darmstümpfe achsial vereinigt. Anfangs hatte der Kranke Neigung zur Diarrhöe, welche gegen Ende der 4 . Woche verschwand. Körpergewichtszunahme um $5 \mathrm{~kg}$. Stuhlgang regelmäßig, ein- bis zweimal täglich. Die Fleischverdauung. war ein wenig gestört und der Fettgehalt des Kotes etwas vermehrt. I Monat später verließ or das Hospital und arbeitete als Schmiedegeselle. Seit Mitte des 4. Monats post operationem fing der Allgemeinzustand an, sich wieder zu verschlechtern: unbestimmte Schmerzen, Gefühl von Völle im Leib, Kurzatmigkeit usw. Es wurden ausgedehnte Geschwulstmetastasen in der Bauchhöhle und an der rechten Lunge konstatiert.

Fall 49. Operateur $\mathrm{Nigris}$ oli. Ein 22jähriger Jüngling, welcher vorher Bauchschmerzen gehabt, klagte über die Zeichen von Darmverschlub. Bei der Laparotomie fand man das Jejunum an einer Stelle verengert und mit dem letzten Ileumabschnitt fest verwachsen. Eine breite Anastomose zwischen der stark erweiterten Jejunumschlinge oberhalb der Striktur und dem ersten Abschnitt des aufsteigenden Colons angelegt und ein $520 \mathrm{~cm}$ langes Stück Dünndarm reseziert. Patient hatte in den ersten Tagen, in denen er nur eine flüssige Nahrung zu sich nahm, Diarrhöe, später durch gemischte Kost Stuhl täglich ein- bis zweimal und breiig, das Körpergewicht nahm rasch zu. Tod $3^{1 / 2}$ Jahre post operationem an tuberkulöser Pleuritis und Perikarditis.

Fall 50. Operateur $\mathrm{G} h \mathrm{ed}$ in i. 44jähriger Arbeiter. Einklem. mung eines Skrotalbruches. Man fand bei der Herniotomie den Darm im Bruchsack gangränös verändert, so wurde ein $20 \mathrm{~cm}$ langes Stück desselben reseziert. Unterdessen kollabierte Patient, und um die Operation schnell zu beenden, wurden die beiden Darmenden an die Wundränder fixiert ohne Anlegung einer Enteroanastomose. 28 Tage später machte man die Bauchhöhle wieder auf, um den Anus praeternaturalis zu beseitigen und fand den zu- sowie abführenden Schenkel mit den anliegenden Darmschlingen mehrfach und unlösbar verlötet, so daß man fast den ganzen Dünndarm, den Blinddarm und das Zweidrittel des aufsteigenden Colons total ausschalten mußte. 
Der Anfangsteil des Jejunums und die obere Partie des Colon ascendens wurden termino-lateral anastomosiert. Schon vom 2. Tage an gingen Stühle durch den natürlichen Weg ab und Kotentlecrungen durch den widernatürlichen After schwanden rom +. Tage an. Stühle waren anfangs flüssig, später breiig und drei- oder viermal täglich. Das Körpergewicht zugenommen. Geheilt entlassen. Zu Hause konnte er als Schuhmacher gut arbeiten. Er hatte täglich einen cin- bis zweimaligen Stuhlgang von breiiger Konsistenz, klagte dann über Diar. rhöe, wenn er mit der Diät nicht sehr vorsichtig war. I I Monate nach der totalen Ausschaltung des Darms erlag er an beiderseitigen krupösen Pneumonic. Bei der Autopsie wies man nach, daß der übrig bleibende Dünndarm bloß $120 \mathrm{~cm}$ lang war, inklusiv des Duodenums. Die Länge des ausgeschalteten Darms konnte man nicht genau bestimmen; Ghedini schätzt dieselbe etwa $534 \mathrm{~cm}$ im ganzen, und zwar $522 \mathrm{~cm}$ rom Dünn- und $12 \mathrm{~cm}$ rom Dickdarm.

Fall 51. Operateur Brenner (zitiert nach Denk). Eine 6rjährige Frau wurde wegen einer eingeklemmten Schenkelhernie operiert. Das schwarzbraun verfärbte $540 \mathrm{~cm}$ lange Stück des Dünndarms wurde resezicrt, das untere Ende des Ileums ins zurückbleibende Jejunum seitlich cingepflanzt und das freie Ende des Jejunums an die Bruchpforte fixiert, um den Darminhalt herauszuleiten. In den näch. sten Tagen kam die Nahrung fast unverdaut und sehr rasch aus dem Jejunalende heraus. Der widernatürliche After wurde allmählich kleiner und schloß sich fast gänzlich $2^{1}$ : . Monate nach der Operation. Der Stuhl erfolgte nur per rectum, war vier- bis fünfmal täglich, dünnflüssig, tonfarbig, höchst übelriechend und enthielt unverdaute Nahrungsbestandteile. Später verlor der Stuhl den üblen Geruch und wurde dicker. $3^{1 / 2}$ Monate post operationem geheilt entlassen. $\mathbf{I}^{1 / 2}$ Jahr nach der Operation befand sich die Kranke vollkommen wohl, war frisch und munter und sorgte für die Hausarbeit ohne Beschwerden. Ihre Nahrung war die von armen Leuten. Appetit gut; Stuhlgang täglich drei- bis viermal, bald breiig, bald mehr flüssig. $2^{1 / 2}$ Jahre post operationem ging sie an zunehmendem Marasmus zugrunde. Bei der Sektion konstatierte man, daß die Länge des übriggebliebenen Dünndarms von der Flcxura duodenojejunalis an gemessen, nur $106 \mathrm{~cm}$ betrug. In diesem Falle wurde somit 83,6 Proz. der gesamten Länge des Jejuno-Ileums reseziert.

Der Übersicht halber fasse ich die oben zitierten 5 I klini. schen Fälle in eine Tabelle kurz zusammen: 


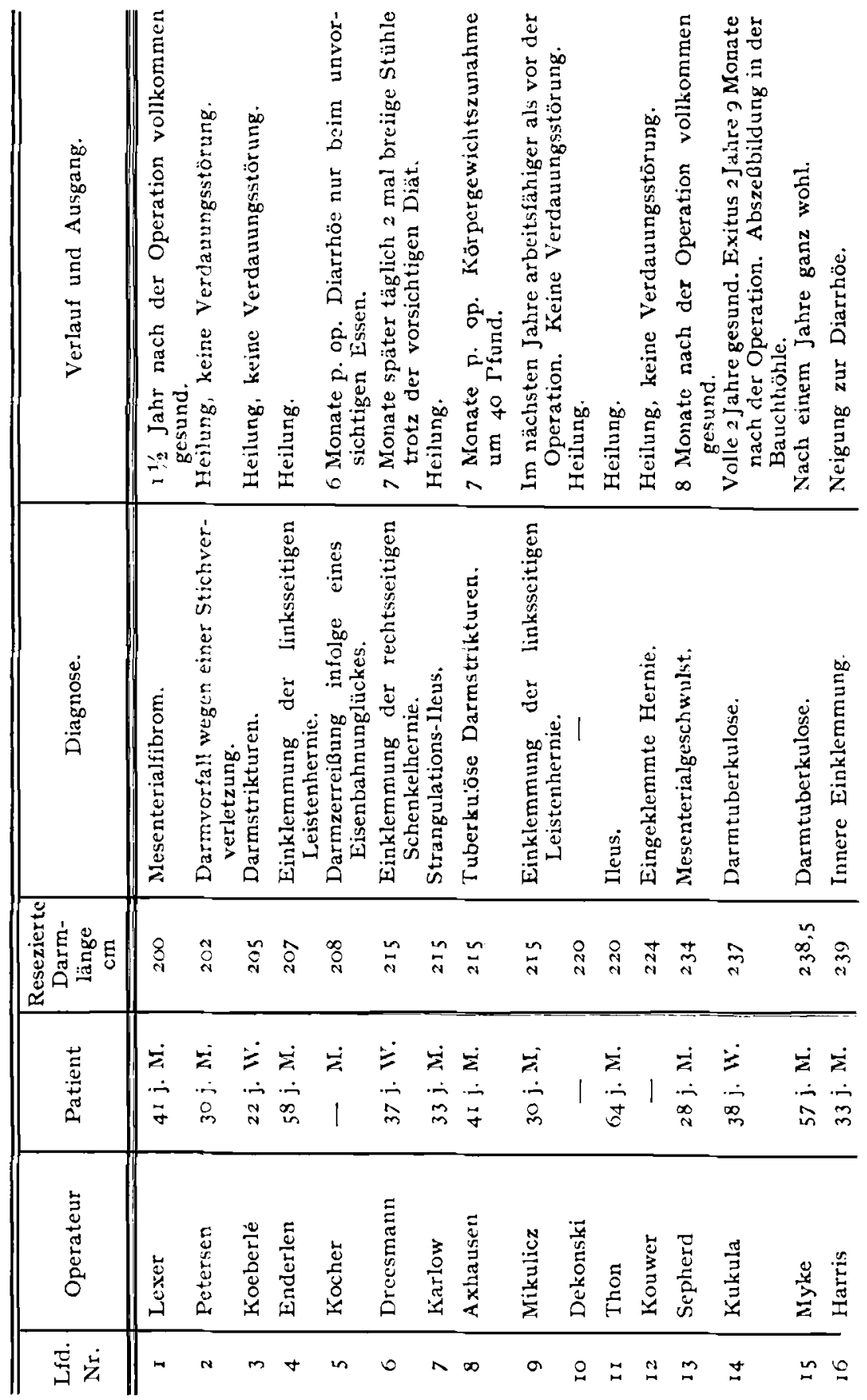




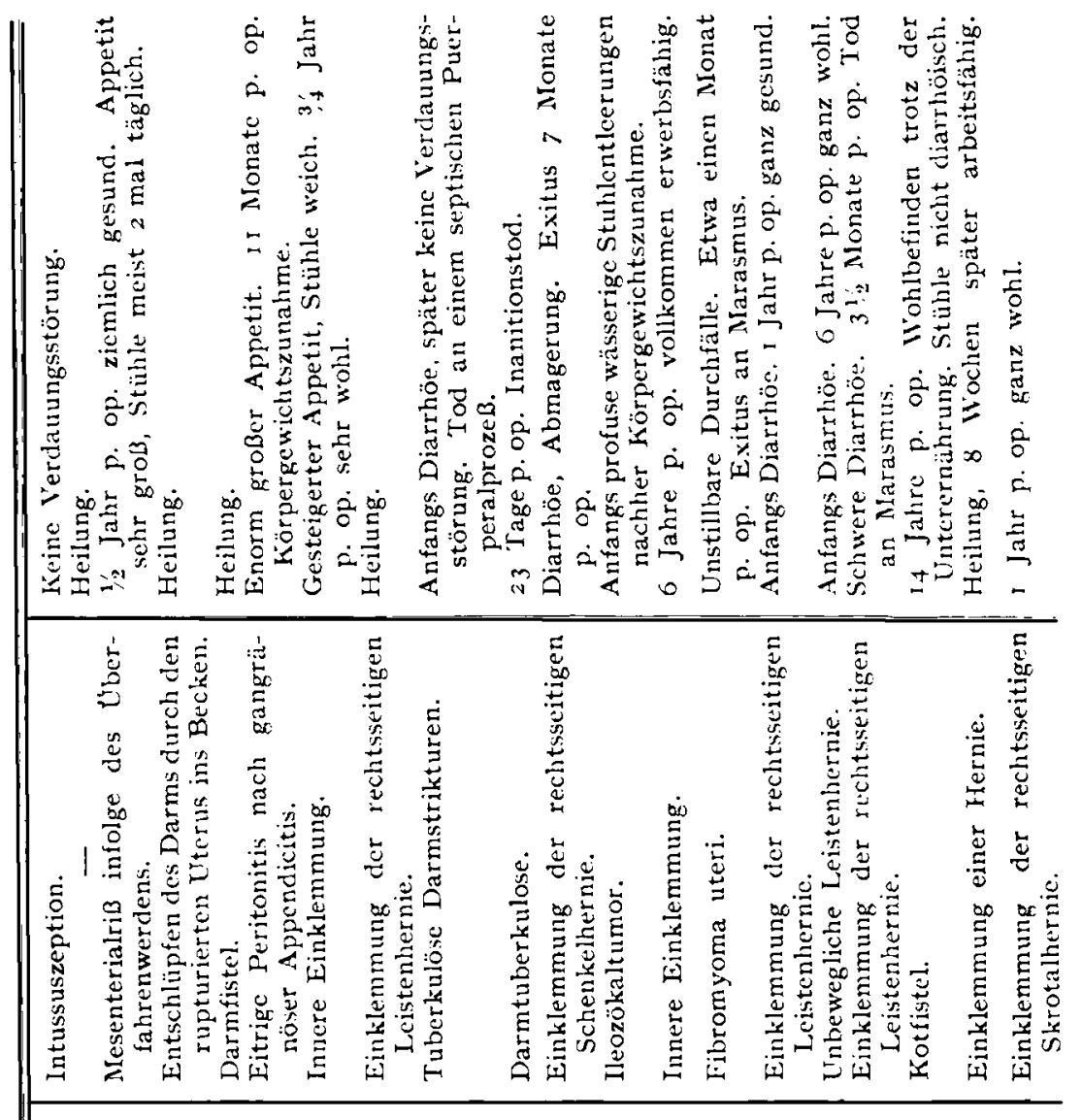

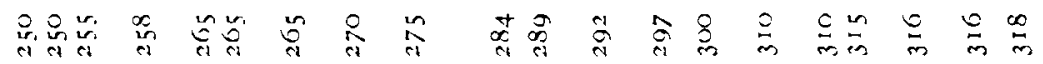

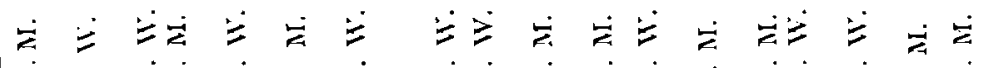

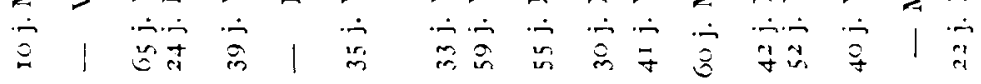

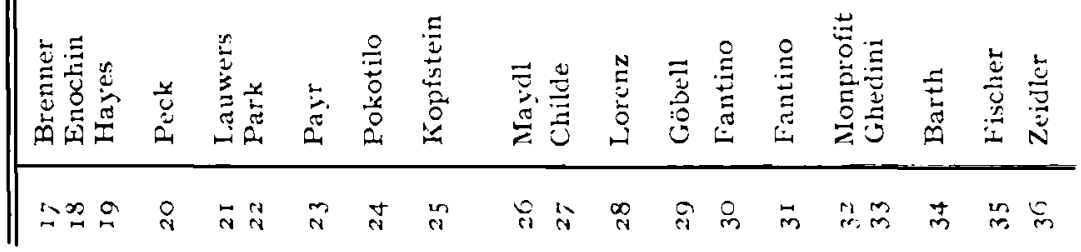




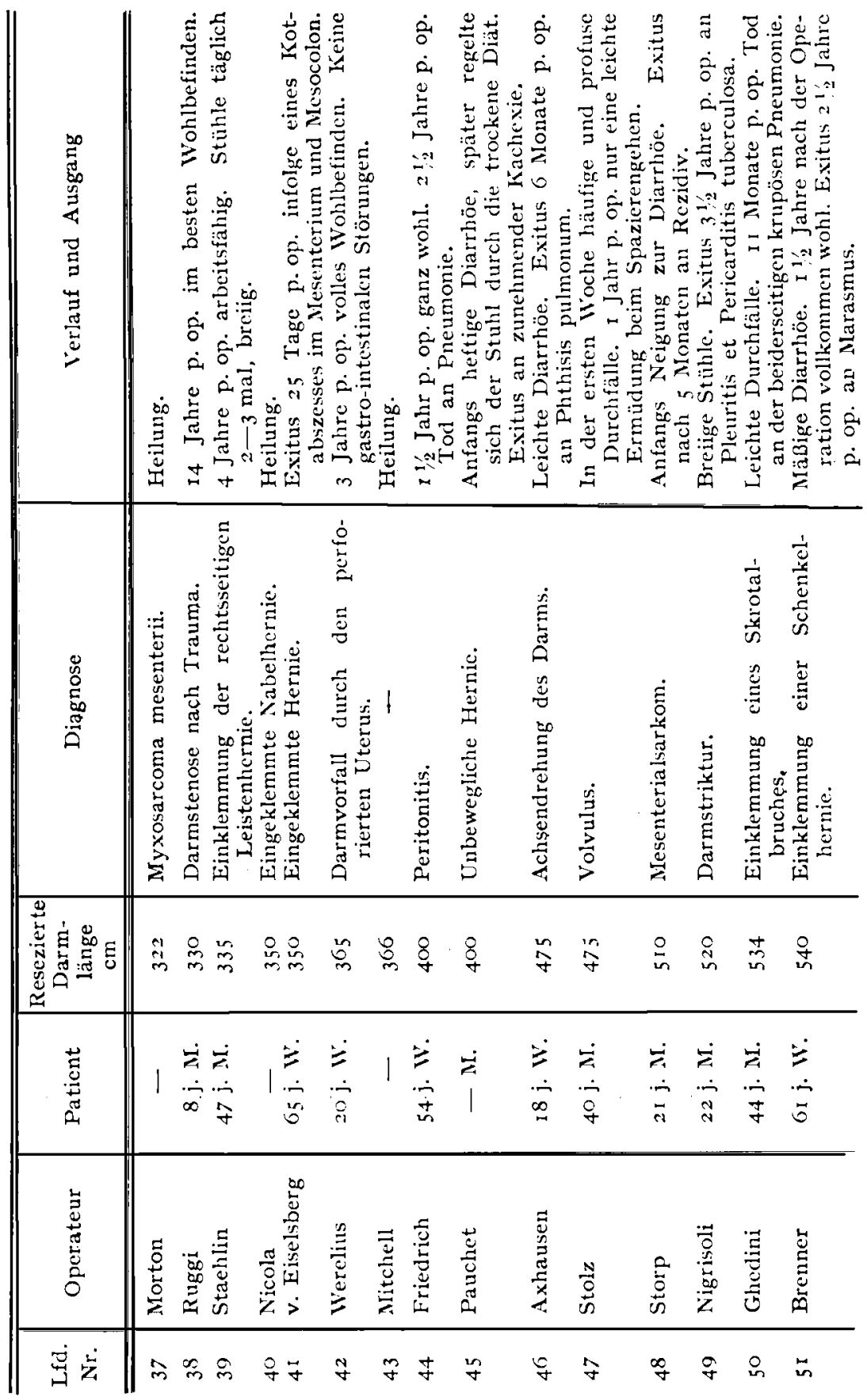


Tierversuche.

Vorbemerkungen. Ich stellte Versuche stets bei Hunden an. Mit den Operationen habe ich so lange gewartet, bis das Gleichgewicht des Stoffwechsels festgestellt wurde. Die sämtlichen Versuchstiere wurden mit ein und derselben Kost gefüttert, welche hauptsächlich aus gekochtem Reis nebst einer geringen Menge von Fisch bzw. Fleisch bestand.

Als schmerzstillendes Mittel injizierte ich $2-5 \mathrm{ccm}$ von I proz. Morphiumlösung unter die Haut, je nach dem Körpergewicht der Hunde, etwa $1 / 2$ Stunde vor der Operation. Die meisten Tiere blieben so ganz ruhig während des ganzen Eingriffes.

Der Hund wurde auf dem Operationstisch in der Rückenlage fixiert. Nachdem der Bauch abrasiert und gehörig desinfiziert war, wurde derselbe in der Mittellinie an der Gegend des Nabels in der Längsrichtung eröffnet. Die Gedärme wurden aus der Wunde herausgezogen. Messung der ganzen Länge des Dünndarms von der Flexura duodenojejunalis bis zum untersten Teil des lleum nach Sappey. Resektion einer bestimmten Länge desselben. Breite seitliche Enteroanastonose nach $\mathrm{H}$. Braun. Wiedermalige Messung des zurückgelassenen Dünndarms, Duodenum dabei nicht mitgerechnet. Reposition desselben in die Bauchhöhle. Schluß der Wunde durch Etagennähte. Bepinselung mit Jodoformkollodium.

Das resezierte Darmstück wurde sofort nach der Operation an der gegenüberliegenden Seite des Mesenterialansatzes in der ganzen Länge aufgemacht, um die Breite und Wanddicke desselben taxieren zu können.

Versuch 1. Weiße Hündin. Körpergewicht 9050 g.

7. XII. 1909. Subkutane Injektion von $2 \mathrm{ccm}$ einer Iproz. Morphiumlösung. Dünndarmlänge $27 \mathrm{I} \mathrm{cm}$. Resektion des unteren Dünndarmabschnittes, $135 \mathrm{~cm}$ lang. Laterale Anastomose. Vom Dünndarm bleibt ein $134 \mathrm{~cm}$ langes Stück ïbrig und zwar rom Jejunum $130 \mathrm{~cm}$ und vom Ileum $4 \mathrm{~cm}$. Breite des resezierten Darms beträgt 28-22 mm, Wanddicke desselben $3-2 \mathrm{~mm}$.

8. XII. Körpergewicht $(=\mathrm{Kgw}$ ) 7900 g. Stühle wäBrig. Appetit fast null. - 9. XII. Kgw. 7500 g. - IO. XII. Kgw. 7200 g. Appetit schlecht. -- II.XII. Kgw. 7 Ioog. - I2. XII. Kgw. $7000 \mathrm{~g}$. 
- 13. XII. Kgw. 6800 g. Etwas munter. - I4. XII. Kgw. 6.550 g. Dünnbreiige Stuhlentleerungen.

I5. XII. Heute Morgen tot gefunden. Deutlich abgemagert. Muskulatur und Fettgewebe stark reduziert. Kein Zeichen von Peritonitis. Magen, Dünn- und Dickdarm sind weder dilatiert noch verdickt, eher etwas verdünnt und sehen sehr bla $B$ aus. Anastomose gut durchgängig. Keine Nahtinsuffizienz. Der zurückgelassene Dünndarm ist $133 \mathrm{~cm}$ lang, und zwar Jejunum $129 \mathrm{~cm}$ und Ileum $4 \mathrm{~cm}$. Breite desselben beträgt 35-30 $\mathrm{mm}$, Wanddicke $1,5-1 \mathrm{~mm}$.

Versuch 2. Schwarz und weiß gefleckte Hündin. Körpergewicht $9950 \mathrm{~g}$.

24. XII. I909. Subkutane Injektion von $2 \mathrm{ccm}$ einer Iproz. Morphiumlösung. Dünndarmlänge $392 \mathrm{~cm}$. Resektion des mittleren Dünndarmabschnittes $290 \mathrm{~cm}$ lang. Laterale Anastomose. Vom Dünndarm bleibt ein $100 \mathrm{~cm}$ langes Stück übrig, und zwar vom Jejunum $30 \mathrm{~cm}$ und vom Ileum $70 \mathrm{~cm}$. Breite des resezierten Darms beträgt 45-35 $\mathrm{mm}$, Wanddicke desselben 3-2 $\mathrm{mm}$.

25. XII. Kgw. $8600 \mathrm{~g}$. Mehrmalige Durchfälle. Appetit schlecht. - 26. XII. Kgw. 8250 g. - 27. XII. Kgw. 8 roo g. Sehr munter, Appetit gebessert. - 28. XII. Kgw. 8500 g. - 29. XII. Kgw. 9100 g. Großer Appetit. - 30. XII. Kgw. 9300 g. Ungewöhnlich starke FreBlust.

3I. XII. Heute Morgen tot gefunden. Nähte der Bauchwand nicht gehalten. Fast sämtliche Baucheingeweide fallen durch die weit klaffende Wunde vor. Kein deutliches Zeichen von Peritonitis. Der zurückgebliebene Dünndarm ist etwa $100 \mathrm{~cm}$ lang und zwar Jejunum $30 \mathrm{~cm}$ und Ileum $70 \mathrm{~cm}$. Keine Nahtinsuffizienz an der Stelle der Anastomose. Magen ist hochgradig dilatiert und voll gefüllt, Dünndarm leer; im Dickdarm befinden sich dünnbreiige chymusartige Massen. Jejunum ist $75-70 \mathrm{~mm}$ breit, $3 \mathrm{~mm}$ dick; Ileum $60-55 \mathrm{~mm}$ breit, $2 \mathrm{~mm}$ dick. Schleimhaut überall katarrhalisch und mit dickschleimigen Massen belegt. Am Ileum hie und da kleine seichte Geschwüre vorhanden.

Versuch 3. Weiß und schwarz gefleckter alter Hund. Körpergewicht I $5400 \mathrm{~g}$.

3I. I. I9Io. Subkutane Injektion von $4 \mathrm{ccm}$ einer I proz. Morphiumlösung. Länge von Jejuno-Ileum $409 \mathrm{~cm}$. Resektion des unteren Dünndarmabschnittes $320 \mathrm{~cm}$ lang. Seitliche Anastomose zwischen Jejunum und Anfangsteil des Dickdarms. Vom Jejunum bleibt ein $8 \mathrm{I} \mathrm{cm} \mathrm{langes} \mathrm{Stück} \mathrm{übrig.} \mathrm{Breite} \mathrm{des} \mathrm{resezierten} \mathrm{Darms} \mathrm{betägt}$ $45-35 \mathrm{~mm}$, Wanddicke desselben $4-3 \mathrm{~mm}$.

I. II. Kgw. 14600 g. Stuhl hart, von normalem Aussehen. Das Tier sieht matt aus. - 2. II. Kgw. I $4400 \mathrm{~g}$. Schwärzliche, blutige, dünnbreiige Stuhlentleerungen. - 3. II. Kgw. I4000 g. - 4. II. Kgw. I3900 g. Appetit gesteigert. - 5. II. Kgw. I3900 g. Blutige, 
wäßrige Diarrhöe. Freßlust stark. - 6. II. Kgw. I3700 g. - 7. II. Kgw. 13350 g. Stuhl teils geformt, teils flüssig. - 8. II. Kgw. I 3200 g. - 9. II. Kgw. 13200 g. - IO. II. Kgw. I2700 g. Körpergewicht nimmt allmählich ab. - Ir. II. Kgw. $12800 \mathrm{~g}$. Stuhl nicht diarrhöisch. - I2. II. Kgw. I3450 g. - 13. II. Kgw. I3500 g. 14. II. Kgw. 13750 g. - 15. II. Kgw. I4 200 g. Stuhl weich, reichlich. - I6. Il. Kgw. I4 I50 g. - 17. II. Kgw. I4 I00 g. - I8. II. Kgw. I4 500 g. - I9. II. Kgw. I4300 g. Profuse Durchfälle. 20. II. Kgw. 13850 g. - 21. II. Kgw. I3 350 g. Stühle weniger profus geworden. - 22. II. Kgw. 14150 g. - 23. II. Kgw. I4250 g. 24. II. Kgw. $14730 \mathrm{~g}$. Heftige Durchfälle. - 25. II. Kgw. I3720 g. -- 26. II. Kgw. I3 800 g. -27 . II. Kgw. I 3600 g. -28 . II. Kgw. I 3400 g. - I. III. Kgw. 13400 g. - 6. III. Kgw. 14850 g. 17. III. Kgw. I 3600 g. -27 . III. Kgw. I5000 g. - I. IV. Kgw. 15250 g. - I5. IV. Kgw. 15250 g. - 21. IV. Kgw. 15300 g. 23. IV. Kgw. $16200 \mathrm{~g}$. -24 . IV. Kgw. $16000 \mathrm{~g} .-$ I. V. Kgw. 16000 g. - I. VI. Kgw. I4400 g. - I. VII. Kgw. I3000 g. I. VIII. Kgw. I3500 g. - I. IX. Kgw. I4600 g. - I. X. Kgw. 14500 g. - I. XI. Kgw. 14500 g. - I. XII. Kgw. I4700 g. - I. I. I9I I Kgw. I3800 g. - I. II. Kgw. I 2600 g. - I. IlI. Kgw II 900 g, - 24. III. Kgw. I $300 \mathrm{~g}$. In den letzten Monaten hat sich der Ernährungszustand deutlich herabgesetzt.

Subkutane Injektion von $4 \mathrm{ccm}$ einer I proz. Morphiumlösung. Eröffnung der Bauchhöhle in der rechten pararektalen Linie. Muskulatur und subkutanes Fettgewebe der Bauchwand stark reduziert. Dünn- und Dickdarm untereinander und mit Omentum majus ziemlich fest verwachsen. Sowohl Dünn- und Dickdarm wie Magen weder dilatiert noch verdickt. Anastomose durchgängig für Daumen.

25. III. Kgw. II 200 g. - I. IV. Kgw. I 000 g. - I 5. IV. Kgw. I I $300 \mathrm{~g}$. -28 . IV. Kgw. i $500 \mathrm{~g}$. In den letzten Tagen hat sich das Tier langsam erholt.

Versuch 4. Schwarzer Hund. Körpergewicht $9800 \mathrm{~g}$.

4. III. 1909. Subkutane Injektion von $4 \mathrm{ccm}$ einer I proz. Morphiumlösung. Länge von Jejuno-Ileum $33 \mathrm{I} \mathrm{cm}$. Resektion des unteren Dünndarmabschnittes $265 \mathrm{~cm}$ lang. Vom Jejunum bleibt ein $60 \mathrm{~cm}$ langes Stück übrig. Der resezierte Darm ist $40-35 \mathrm{~mm}$ breit, 3,5 bis $2,5 \mathrm{~mm}$ dick.

5. III. Kgw. 8750 g. Breiige Stühle. - 6. III. Kgw. 8200 g. Appetit minimal. - 7. III. Kgw. 7800 g. - 8. III. Kgw. 7700 g. Blutige Diarrhöe. - 9. III. Kgw. $7350 \mathrm{~g}$. Fast keine Freßlust. I0. III. Kgw. 7150 g. - II. III. Kgw. 7 I00 g. Heute Mittag Tod. Stark abgemagert. Kein Zeichen von Peritonitis. Gedärme etwas kollabiert, mit verdünnter Wandung. Anastomose nicht stenosiert. Drüsige Bauchorgane anämisch. Länge des zurückgelassenen Dünndarms von der Flexura duodenojejunalis bis zur Stelle der Anastomose, 
dicht oberhalb der Valvula Bauhini beträgt $6 \mathrm{I} \mathrm{cm}$, Breite des Jejunums 45-40 mm, Wanddicke desselben 3,5-3,0 mm. Beide Lungen von zahllosen miliargroßen gelblichgrauen Tuberkeln durchsetzt.

Versuch 5. Fuchsfarbig und weiB gefleckter Hund. Körpergewicht $19750 \mathrm{~g}$.

23. III. 19IO. Subkutane Injektion von $4 \mathrm{ccm}$ einer I proz. Morphiumlösung. Länge von Jejuno-Ileum $412,5 \mathrm{~cm}$. Resektion desselben $335 \mathrm{~cm}$ lang. Seitliche Anastomose. Vom Dünndarm bleibt ein $75 \mathrm{~cm}$ langes Stück übrig, und zwar vom Jejunum $61 \mathrm{~cm}$ und rom lleum $14 \mathrm{~cm}$. Breite des resezierten Darms beträgt $40-35 \mathrm{~mm}$, Wanddicke desselben $3,0-2,5 \mathrm{~mm}$.

24. III. Kgw. I 8200 g. - 25. III. Kgw. 17500 g. Etwas blutige Diarrhöe. - 26. III. Kgw. I7100 g. - 27. III. Kgw. I6650 g. Wässerige Stühle. - 28. III. Kgw. 16 I00 g. - 29. III. Kgw. I5500 g. -30 . III. Kgw. 15200 g. - 31. III. Kgw. 15200 g. - I. IV. Kgw. 15000 g. -2 . IV. Kgw. I $455^{\circ}$ g. - 3. IV. Kgw. I4500 g. - 4. IV. Kgw. 13900 g. - 5. IV. Kgw. 13600 g.

6. IV. Heute Morgen tot gefunden. Hochgradig abgemagert, beinahe skelettiert. An der linken Hypogastrialgegend befindet sich ein ausgedehnter subkutaner AbszeB. Thoraxorgane intakt. Kein Zeichen von Peritonitis. Der zurückgebliebene Dünndarm ist $72,5 \mathrm{~cm}$ lang, und zwar Jejunum $58,5 \mathrm{~cm}$ und Ileum $14 \mathrm{~cm}$. Duodenum ist $60 \mathrm{~mm}$ breit, $3 \mathrm{~mm}$ dick, Jejunum $60-55 \mathrm{~mm}$ breit, $3,0-2,5 \mathrm{~mm}$ dick, Ileum $50-35 \mathrm{~mm}$ breit, $2,5 \mathrm{~mm}$ dick und Dickdarm $70-50-70 \mathrm{~mm}$ breit, $2,5 \mathrm{~mm}$ dick.

Versuch 6. Brauner Hund. Körpergewicht $14400 \mathrm{~g}$.

2. II. I910. Subkutane Injektion von $4 \mathrm{ccm}$ einer 1 proz. Morphiumlösung. Länge von Jejunum und Ileum $520 \mathrm{~cm}$. Von der Fexura duodenojejunalis an analwärts wird ein $435 \mathrm{~cm}$ langes Stück Dünndarm reseziert. Nach der Anastomosenbildung bleibt ein $80 \mathrm{~cm}$ langer Teil Ileum übrig. Breite des resezierten Darms beträgt 45 bis $30 \mathrm{~mm}$, Wanddicke desselben 3-2 $\mathrm{mm}$.

3. II. Kgw. 13000 g. - Munter. Freßlust nicht besonders vermindert. - 4. II. Kgw. I2 800 g. Stuhl nicht diarrhöisch. Appetit enorm groß. - 5. II. Kgw. 12250 g. - 6. II. Kgw. I 2100 g. - 7. II. Kgw. 11800 g. -- 8. II. Kgw. I I 900 g. - 9. II. Kgw. I 2000 g. IO. II. Kgw. 12000 g. - II. II. Kgw. II 800 g. Fester, geformter Kot. - 12. I1. Kgw. II 600 g. - 13. II. Kgw. I 250 g, Leichte Diarrhöe. - I4. II. Kgw. I 250 g. Appetit vermindert. - I5. II. Kgw. I 1000 g. Allmähliche Abmagerung. Haare ohne Glanz. 16. II. Kgw. I 000 g. Stühle breiig, reichlich. Appetit etwas gesteigert. - I7. II. Kgw. I 900 g. - I8. II. Kgw. I 2000 g. Seit einigen Tagen hat sich das Tier etwas erholt. - I9. II. Kgw. $12060 \mathrm{~g}$. - 20. II. Kgw. $10250 \mathrm{~g}$. Ziemlich rapide Abnahme des Körpergewichtes. - 2I. II. Kgw. 9900 g. Stühle blutig, nicht reichlich. 
Freßlust wieder vermindert. - 22. II. Kgw. 9800 g. - 23. II. Kgw. $10100 \mathrm{~g}$. - 24. II. Kgw. $10000 \mathrm{~g}$. Appetit ein wenig besser. 25. II. Kgw. 10000 g. -26 . II. Kgw. 9800 g. -27 . II. Kgw. 9900 g. - 28. II. Kgw. 9700 g. - I. III. Kgw. 9500 g. - 3. III. Kgw. 9700 g. Freßlust minimal. Leichte Diarrhöe. - 5. III. Kgw. 9700 g. - 8. III. Kgw. 9300 g. - I4. III. Kgw. 9 I 50 g. Dünnbreiige Stühle. Großer Kräfteverlust. Gang kaum möglich. - 15. III. Kgw. $8600 \mathrm{~g}$. - 16. III. Kgw. 8450 g. - 17. III. Kgw. 8400 g.

I8. III. Heute Mlorgen tot gefunden. Stark abgemagert. Subkutanes Fettgewebe beinahe verschwunden. Auch Muskulatur stark reduziert. MIagen ziemlich deutlich dilatiert, Wandung desselben schlaff und dünn. Länge des Dünndarms von der Anastomose an analwärts $92 \mathrm{~cm}$. An der Stelle $20 \mathrm{~cm}$ oralwärts von der Ileocöcalklappe befindet sich eine Invagination; Lumen des Darmes ist daselbst stark verengert, für einen Bleistift durchgängig. Breite des Duodenums vom Pylorus bis zur Anastomose beträgt $80-70 \mathrm{~mm}$, Wanddicke desselben 2,5-2,0 mm, Breite desDünndarms von der Anastomose bis zur Intussusception $70-55 \mathrm{~mm}$, Wanddicke desselben 2,5 bis $I, 5 \mathrm{~mm}$, und Breite des Dünndarms ron der Invagination bis zur Valvula Bauhini $40-30 \mathrm{~mm}$, Wanddicke desselben $2,5 \mathrm{~mm}$. Schleimhaut des ganzen Darmtraktus katarrhalisch verändert, beinahe faltenlos. An der Rektalschleimhaut findet man ziemlich zahlreiche kleine Hämorrhagien.

Versuch 7 . Weiß und schwarz gefleckter Hund. Körpergewicht $16000 \mathrm{~g}$.

2. IX. 1910. Erste Operation. Subkutane Injektion von $4 \mathrm{ccm}$ einer I proz. Morphiumlösung. Ganze Länge von Jejuno-Ileum $374 \mathrm{~cm}$. Rescktion desselben $327 \mathrm{~cm}$ lang. Nach der Anastomosenbildung blcibt cin $32 \mathrm{~cm}$ langes Stück Jejunum und ein $13 \mathrm{~cm}$ langes Stück Ileum übrig. Breite des resezierten Darms mißt $45-35 \mathrm{~mm}$, Wanddicke desselben $3-2 \mathrm{~mm}$.

3. IX. Kgw. $14200 \mathrm{gr}$. Leichte Diarrhöe. Freßlust nicht besonders vermindert. - 4. IX. Kgw. $13800 \mathrm{~g}$. Flüssige, etwas blutige Durchfälle. - 5. IX. Kgw. 13700 g. - 6. IX. Kgw. I3 500 g. - 7. IX. Kgw. $13100 \mathrm{gr}$ - 8. IX. Kgw. I3150 g. Stühle dunnbreiig. 9. IX. Kgw. $13200 \mathrm{~g}$. - I0. IX. Kgw. $13100 \mathrm{~g}$. - II. und I2. IX. Kgw. 13200 g. Appetit vermehrt. - I3. IN. Kgw. 13250 g. -. I4. und 15. IX. Kgw. $13300 \mathrm{~g}$. - I6. IX. Kgw. $13250 \mathrm{~g}$. - 17. IX. Kgw. $13300 \mathrm{~g} .-18 . \mathrm{IX}$. Kgw. $13350 \mathrm{~g} .-2$ I. IA. Kgw. I3 $400 \mathrm{~g} .-$ 24. IX. Kgw. 13600 g. - 27. IX. Kgw. 13600 g. - 1. A. Kgw. 13750 g. -15. X. Kgw. I3950 g. -25 . X. Kgw. 14000 g. - I. XI. Kgw. 13200 g. - 15. XI. Kgw. i 3650 gr. - I. XII. Kgw. I+200 g. 15. XII. Kgw. I 200 g. - I. I. I9I Kgw. $1+300$ g. - II. I. Kgw. I $4600 \mathrm{~g} .-12$. I. Kgw. I $4600 \mathrm{~g}$. 
Zweite Operation. Subkutane Injektion von $4 \mathrm{ccm}$ einer I proz. Morphiumlösung. Laparotomie. Länge des zurückgelassenen JejunoIleums beträgt $44 \mathrm{~cm}$. Magen und Dünndarm sehr deutlich erweitert. Der Dünndarm von der Flexura duodenojejunalis an bis zum untersten Teil vom Ileum nebst Wurmfortsatz gänzlich entfernt, beide Darmenden an und für sich geschlossen und eine seitliche Anastomose zwischen dem Duodenum, $5 \mathrm{~cm}$ oralwärts von der Flexura duodenojejunalis und dem Anfangsteil des Dickdarms angelegt. Breite des resezierten Darmabschnittes 70-65 $\mathrm{mm}$, Wanddicke desselben durchschnittlich $4 \mathrm{~mm}$.

13. I. Kgw. 14000 g. Stark abgeschwächt. - I4. I. Kgw. I3500 g. Appetil gering. - 15. I. Kgw. 13500 g. - 16. I. Kgw. 12800 g. FreBlust beinahe verloren. - 17. I. Kgw. $12500 \mathrm{~g}$.

I8. I. Heute Morgen tot gefunden. Stark abgemagert, subkutanes Fettgewebe hochgradig reduziert. Kein Zeichen von Peritonitis. Magen nicht so deutlich dilatiert wie bei der zweiten Operation. Duodenum $70 \mathrm{~mm}$ breit, $4-5 \mathrm{~mm}$ dick. Schleimhaut geglättet und katarrhalisch. Dickdarm $45 \mathrm{~mm}$ breit, $2 \mathrm{~mm}$ dick, Falten der Schleimhaut erhalten. Unzählige alte kleine submuköse Blutungen.

Versuch 8. Brauner Hund. Körpergewicht $20300 \mathrm{~g}$.

I9. VIII. IgIo. Subkutane Injektion von $5 \mathrm{ccm}$ einer $\mathrm{s}$ proz. Morphiumlösung. Länge des Jejuno-Ileums $450 \mathrm{~cm}$. Resektion desselben $400 \mathrm{~cm}$ lang. Seitliche Anastomose. Der übrig bleibende Dünndarm ist $45 \mathrm{~cm}$ lang und zwar Jejunum $35 \mathrm{~cm}$ und Ileum $10 \mathrm{~cm}$. Breite des resezierten Dünndarms beträgt $43-35 \mathrm{~mm}$, Dicke desselben $3,5-2,5 \mathrm{~mm}$.

20. VIII. Kgw. 19100 g. - 2I. VIII. Kgw. 19000 g. - 2. VIII. Kgw. 18800 g. - 23. VIII. Kgw. 18600 g. - 24. VIII. Kgw. 18000 g. - 25. VIII Kgw. I8000 g. - 26. VIII. Kgw. 18100 g. -27 . VIII. Kgw. 18100 g. -28 . VIII. Kgw. I 8100 g. -29 . VIII. Kgw. 88150 g. - 30. VIII. Kgw. 18200 g. - I. IX. Kgw. I8 I00 g. - 2I. IX. Kgw. 17450 g. - 1.X. Kgw. 17800 g. - I. XI. Kgw. 18000 g. - I. XII. Kgw. 18000 g. - I. I. I9I I Kgw. I7900 g. -- I. II. Kgw. I7800 g. - I. III. Kgw. 18000 g. - I5. III. Kgw. I 8150 g. - 24. III. Kgw. I $8400 \mathrm{~g}$.

Laparotomie. Magen, Dünn- und Dickdarm, besonders die beiden ersteren stark dilatiert, die Wandungen sehr verdickt, etwa zweifach so dick wie bei der ersten Operation. Hie und da findet man fibrinöse Adhäsionen zwischen dem großen Netz und den Darmschlingen. Länge des Jejuno-Ileums beträgt $44 \mathrm{~cm}$. Schluß der Bauchwunde.

25. III. Kgw. 18000 g. - 26. III. Kgw. 18600 g. -27 . III. Kgw. I8700 g. - 28. III. Kgw. I8 800 g. - 29. III. Kgw. I8600 g. - 30. und 3I. III. Kgw. I 8500 g. - I. IV. Kgw. I 8400 g. -28 . IV. Kgw. $18500 \mathrm{~g}$. 
Zwar tritt zeitweise eine leichte Diarrhöe auf, jedoch wird das Tier dadurch nicht besonders angegriffen.

Versuch 9. Fuchsfarbiger Hund. Körpergewicht $8100 \mathrm{~g}$.

23. III. 1910. Subkutane Injektion ron $3 \mathrm{ccm}$ einer 1 proz. Morphiumlösung. Länge des Jejuno-lleums $350 \mathrm{~cm}$. Resektion des unteren Abschnittes desselben $315 \mathrm{~cm}$ lang. Nach der Anastomosenbildung bleibt rom Dünndarm ein $30 \mathrm{~cm}$ langes Stück Jejunum übrig. Breite des resezierten Darms beträgt to--30 $\mathrm{mm}$, Wanddicke desselben 3-2 $\mathrm{nm}$.

24. III. Kgw. 7350 g. -- 25. III. Kgw. 7000 g. Blutige Durchfälle. 26. III. Kgw. $; 000 \mathrm{~g}$. -27 . III. Kgw. 6y00 g. - 28. III. Heute Morgen tot gefunden. Stark abgemagert. Subkutanes Fettpolster beinahe verschwunden. Kein Zeichen von Peritonitis. Magen inhaltlos. Der zurückgelassene Dünndarm ist nur $30 \mathrm{~cm}$ lang. Breite des Darms rom Pylorus bis zur Stelle der Anastomose beträgt duchschnittich $40 \mathrm{~mm}$. Wanddicke desselben Teils etwa $3 \mathrm{~mm}$, Breite des Darms von der Stelle der Anastomose bis zur Ampulla recti $30 \mathrm{~mm}$. Wanddicke desselben Teils $2 \mathrm{~mm}$. Am unteren Abschnitte des Dickdarms sieht man viele kleine petechienartige Schleimhaut. blutunger.

Versuch Io. Schwarzer Hund. Körpergewicht $13900 \mathrm{~g}$.

22. VII. I9Io. Subkutane Injektion von $3 \mathrm{ccm}$ einer 1 proz. Morphiumlösung. Länge des Jejuno-Ileums $504 \mathrm{~cm}$. Resektion des Dunndarms $485 \mathrm{~cm}$ lang. Der übrigbleibende Dünndarm ist $13,5 \mathrm{~cm}$ lang, und zwar Jejunum $6.5 \mathrm{~cm}$ und Ileum $7 \mathrm{~cm}$. Breite des resezierten I)arms beträgt to-32 $\mathrm{mm}$ und Wanddicke desselben 3,5 bis $2.5 \mathrm{~mm}$.

23. VII. Kgw. I $2800 \mathrm{gr}-24$ VII. Kgw. $12500 \mathrm{gg}-25$. VIl. Kgw. 12500 g. -26 . VII. Kgw. I2 400 g. -27 . VII. Kgw. 12550 g. -28 . und 29. VII. Kgw. $12600 \mathrm{~g}$. 30. VII. Kgw. $12700 \mathrm{~g}$. 31. VII. Kgw. $12600 \mathrm{~g}$. - 1. V1II. Kgw. 12700 g. - 2. und 3. VIII. Kgw. $12600 \mathrm{~g}$. - 4. VIII. Kgw. $12500 \mathrm{~g}$. - 5. VIII. Heute tot gefunden. Kein Zeichen von Bauchfellentzündung. Anastomose gut durchgängig. Weder Dilatation noch Wandverdickung des zurückgelassenen I)arms, Auch Magen nicht erweitert, Wandung desselben etwas schlaff.

Versuch II. Weiß und schwarz gefleckter $\mathrm{H}$ und. Körpergewicht $13100 \mathrm{~g}$.

I. VIII. I9Io. Subkutane Injektion ron $3 \mathrm{ccm}$ einer 1 proz. Morphiumlösung. Resektion des ganzen Jejunums und Ileums. Laterale Anastomose zwischen I) uodenum, $+\mathrm{cm}$ oralwärts von der Flexura duodenojejunalis und Anfangsteil des Dickdarms, $2 \mathrm{~cm}$ analwärts von der Valvula Bauhini. 
2. VIII. Kgw. I 750 g. - 3. VIII. Kgw. I 700 g. Profuse Durchfälle. - 4. VIII. Kgw. II 500 g. - 5. VIII. Heute Morgen tot gefunden. Kein Zeichen von Peritonitis. Keine makroskopisch nachweisbare Veränderung am ganzen Darmtraktus.

Fassen wir die oben erwähnten Resultate der Tierversuche kurz zusammen, so hatten die Versuchstiere in der Regel bald nach der Operation profuse Durchfälle. Dieselben wurden später allmählich seltener, sistierten jedoch nicht ganz. Im Gegenteil kehrte die Diarrhöe von Zeit zu Zeit zurück, dadurch wurde der inzwischen etwas gehobene Ernährungszustand wieder herabgesetzt.

5 Hunde (Versuch $I, 4,9$, 10 und 11 ), an denen die Operation zu eingreifend war, sahen darnach ganz kraftlos aus. Sie verloren ihre Freßlust beinahe gänzlich. Wegen der verminderten Nahrungsaufnahme einerseits, der profusen Durchfälle andererseits magerten sie fast bis zum Skelett ab und gingen schließlich unter den Symptomen einer hochgradigen Inanition zugrunde.

6 andere Hunde (Versuch 2, 3, 5, 6, 7 und 8) erholten sich nach der Operation trotz einer heftigen Diarrhöe. Ihr Appetit war ein sehr großer. Sie fraßen anfangs alle Speisearten ohne Wahl, später zogen sie vegetabilische Nahrung vor.

Unter diesen 6 Hunden starb I (Versuch 2, Resektion 74,5 Proz.) am 8. Tage am Klaffen der Operationswunde, ein anderer (Versuch 5, Resektion 82 Proz.) am 15. Tage an einer ausgedehnten AbszeBbildung, und ein 3. (Versuch 6, 85 Proz.) am 45. Tage an der chronischen Invagination. Unter den 3 übrigen lebt I (Versuch 3, Resektion 80 Proz.) I 5 Monate nach der Operation, ein 2. (Versuch 7 , Resektion 87 Proz.) über 4 Monate bis zur 2. Operation und endlich ein 3. (Versuch 8, Resektion go Proz.) über 8 Monate in einem ziemlich guten Ernährungszustande.

Bei der Sektion bzw. Probelaparotomie konstatierte man bei den letzteren 6 Hunden, die die Operation gut überstanden, eine ziemlich deutliche kompensatorische Hypertrophie des Magens und Darms, besonders auffallend in Versuchen 7 und 8 , während man an den ersteren 5 Hunden, welche der Operation zum Opfer fielen, weder Dilatation noch Wandverdickung der Gedärme nachweisen konnte.

Ich stellte noch Experimente an 7 weiteren Hunden an. 5 davon (Resektion 50, 60, 80, 87 bzw. 88 Proz.) erlagen an einer Peritonitis am 6. bis i 1. Operationstage. Ein 6. (Resektion 60 Proz.) starb an Pneumonie am Ende der 2. Woche und ein 7. (Resektion 84 Proz.) an Chok während der Operation.

Um die obigen Versuche übersichtlich zu machen, fassse ich sie tabellarisch zusammen (siehe S. 460--46I). 


\section{Schlußbetrachtung.}

I. Bei einer ausgedehnten Darmresektion läßt sich die Schwere des Eingriffs besser an der relativen Länge des resezierten Dünndarmstückes erkennen als an der absoluten, da die Gesamtlänge des Jejuno-Ileum individuell sehr variabel ist.

2. Brenners Fall, in welchem 83,6 Proz. Dünndarm reseziert wurde, ist wohl die ausgedehnteste, mit Erfolg ausgeführte Darmresektion unter den bis jetzt publizierten Resektionsfällen.

3. Beim Hunde kann man unter günstigen Bedingungen noch mehr, und zwar bis zu 90 Proz. der ganzen Länge des Jejuno-Ileum ohne großen Nachteil resezieren.

4. Aus den klinischen Erfahrungen und experimentellen Ergebnissen glaube ich annehmen zu dürfen, daß die Darmresek. tion etwa bis zu 80 Proz. der ganzen Länge des Jejuno-lleum beim Menschen ein zulässiger Eingriff sei.

5. Eine kompensatorische Hypertrophie oder Dilatation des zurückgelassenen Darms läßt sich an denjenigen Tieren meist nachweisen, welche die Operation gut überstanden haben; in den Fällen hingegen, welche den Eingriff nicht lange überleben konnten, in der Regel nicht.

6. Unterschiede in bezug auf die Prognose der Operation je nach den Abschnitten des resezierten Darms sind nicht konstatierbar.

Zum Schluß sei mir erlaubt, unserem Chef, Herrn Prof H. I to, für sein förderndes Interesse an der vorliegenden Arbeit meinen besten Dank auszusprechen. 


\begin{tabular}{|c|c|c|c|c|c|}
\hline 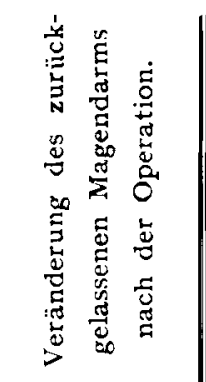 & 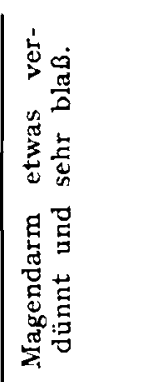 & 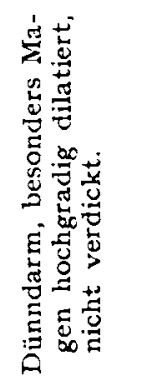 & 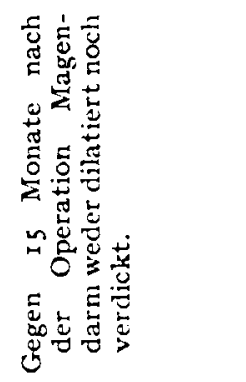 & 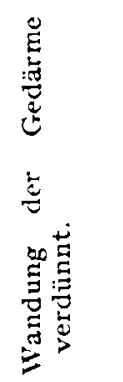 & 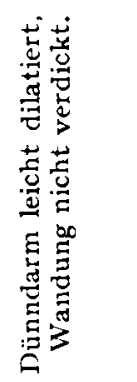 \\
\hline 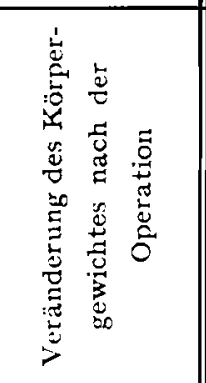 & 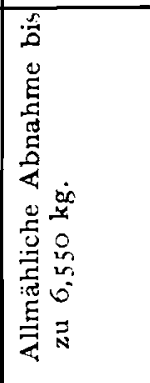 & 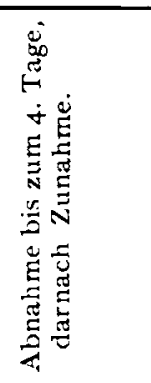 & 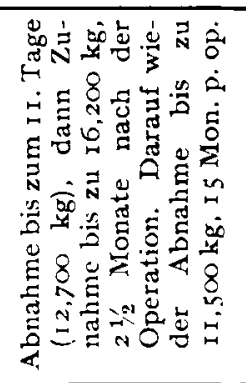 & 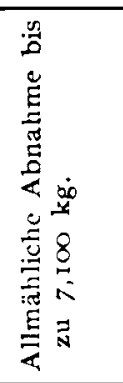 & 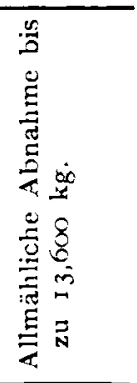 \\
\hline 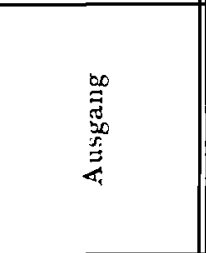 & 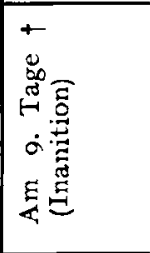 & 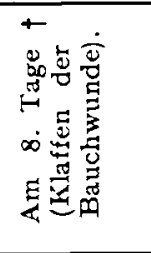 & 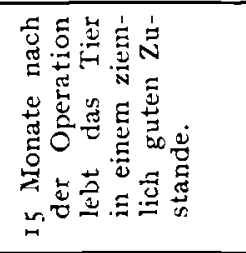 & 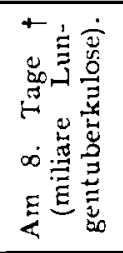 & 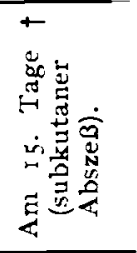 \\
\hline 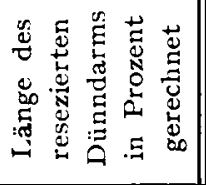 & ir. & $\stackrel{n}{+}$ & $\stackrel{\infty}{\infty}$ & $\frac{3}{\infty}$ & $\hat{\infty}$ \\
\hline 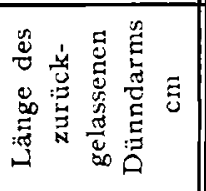 & 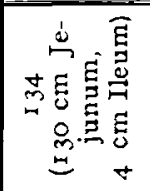 & 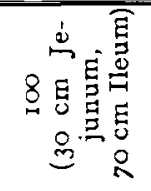 & 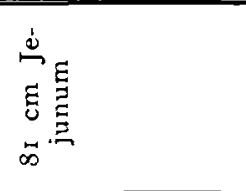 & $\stackrel{\stackrel{\dot{S}}{E}}{E} \underset{g}{E}$ & 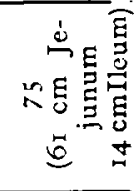 \\
\hline 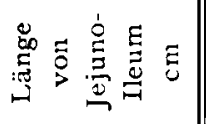 & ลิ) & Sू & $\hat{q}$ & $\approx$ & $\begin{array}{l}\stackrel{i r}{c} \\
\stackrel{+}{+}\end{array}$ \\
\hline 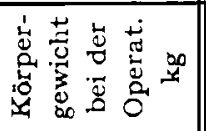 & $\begin{array}{l}0 \\
0 \\
0 \\
0 \\
0\end{array}$ & $\begin{array}{l}0 \\
2 \\
0 \\
0 \\
0\end{array}$ & $\begin{array}{l}8 \\
8 \\
i n \\
n \\
n\end{array}$ & $\underset{0}{8}$ & م. \\
\hline 离 & i & $\dot{s}$ & 马્ય & $\vec{z}$ & $>$ \\
\hline
\end{tabular}


Experiment. u. literar. Studien über d. ausgedehnten Dünndarmresektionen. 46 I

\begin{tabular}{|c|c|c|c|c|c|c|}
\hline 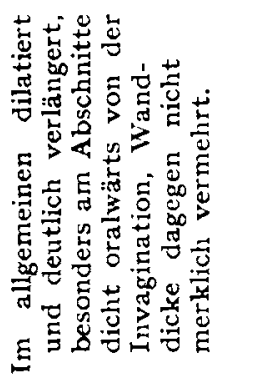 & 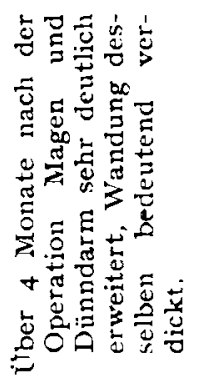 & 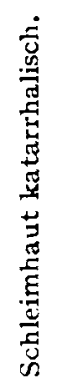 & 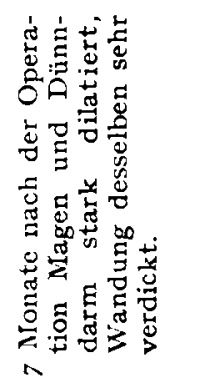 & 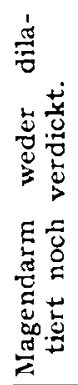 & 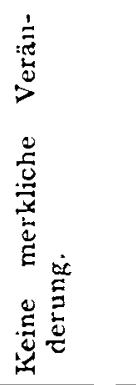 & 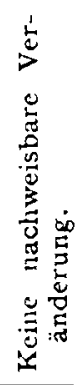 \\
\hline 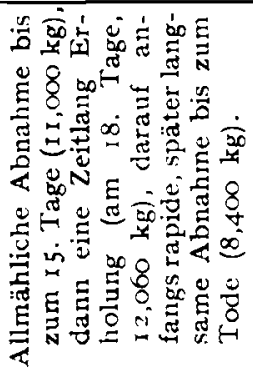 & 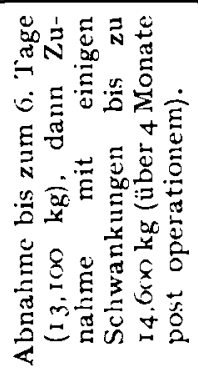 & 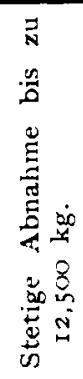 & 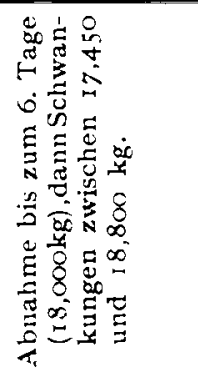 & 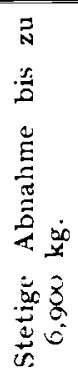 & 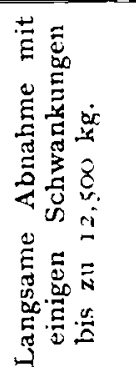 & 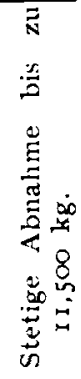 \\
\hline 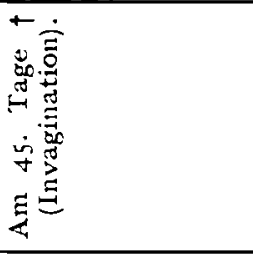 & 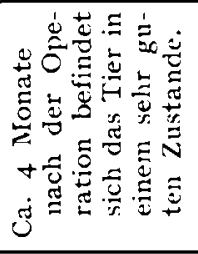 & 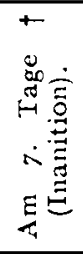 & 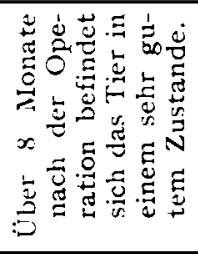 & 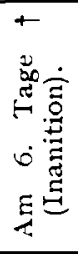 & 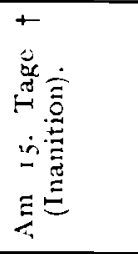 & 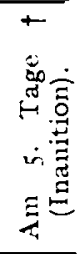 \\
\hline$\infty$ & $\infty$ & $\bar{\Xi}$ & 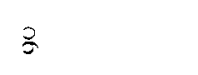 & $\underset{\Xi}{\Xi}$ & s & 8 \\
\hline 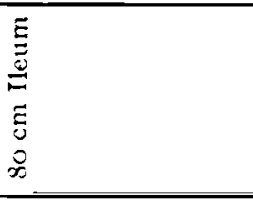 & 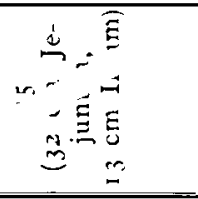 & & 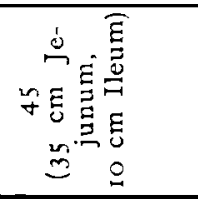 & 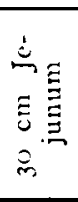 & 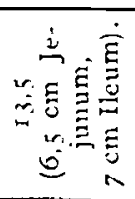 & 1 \\
\hline $\begin{array}{l}3 \\
\text { in }\end{array}$ & 芯 & 1 & $\stackrel{0}{m}$ & $\stackrel{2}{2}$ & $\underset{0}{+}$ & 1 \\
\hline $\begin{array}{l}8 \\
+ \\
\dot{y}\end{array}$ & $\begin{array}{l}8 \\
\vdots \\
\vdots \\
\vdots \\
0\end{array}$ & $\begin{array}{l}8 \\
8 \\
\\
\end{array}$ & 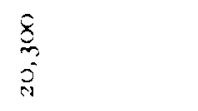 & $\frac{8}{\infty}$ & $\underset{8}{\stackrel{8}{8}}$ & $\frac{g}{2}$ \\
\hline 5 & 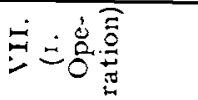 & $\begin{array}{l}-\dot{0} \\
=\dot{0}\end{array}$ & $\stackrel{\Xi}{\rightleftharpoons}$ & $\ddot{x}$ & $\dot{x}$ & $\ddot{V}$ \\
\hline
\end{tabular}




\section{Literaturverzeichnis.}

I. A 1 b u , A., Utber die Grenzen der Zulässigkeit ausgedehnter Darmresektionen. Berliner klinische Wochenschrift. I90I, Nr. 50, S. 1248.

2. Derselbe, Versuche über Ausschaltung von Dünn- und Dickdarm. Mitteilungen aus den Grenzgebieten der Medizin und Chirurgie 1909, Bd. I9, S. 852 .

3. De r s e I b e , Bemerkungen zu der Erwiderung des Herrn Dr. Denk in Bd. 20 , Heft 2, gegen meine Arbeit ,Versuche über Ausschaltung von Dünn- und Dickdarm" in Bd. I9, Heft 5. Ebendaselbst I910, Bd. 21, S. I 79.

4. A $x \mathrm{~h}$ a u s e n, G., Zur Frage der sogenannten akuten postoperativen Magendilatation nebst Bemerkungen zur Technik der hinteren Gastroenterostomie. Deutsche mediz. Wochenschr. 1909, Nr. 4, S. I 45.

5. Ders e l b e, Úber die obere Grenze für die Zulässigkeit ausgedehnter Dünndarmresektionen. Mitteilungen aus den Grenzgebieten der Medizin und Chirurgie I910, Bd. 21, S. 55 .

6. Barke r, Arthur E. J., Three cases illustrating the condition of the small intestine some years after extensive enterectomies. The Lancet. I905, April 22, S. 1062 .

7. B a u m, W., Wie viel Darm kann der Mensch entbehren? Fortschritte der Medizin I884, Bd. 2, S. 793.

8. Be neke, Über die Länge des Darmkanals bei Kindern, sowie über die Kapazität des Magens Neugeborener. Deutsche mediz. Wochenschr. I880, Nr. 32 und 33, S. 433 und 448.

9. B la $y$ n e y, A le $x$ a nder, On the removal of great lengths of intestine. The British Medical Journal 1901, Vol. II, Nov. 16, S. 1456.

10. Braun, He in $\mathbf{r}$ ich, Uber die Enteroanastomose als Ersatz der zirkulären Darmnaht. Archiv für klin. Chir. 1893, Bd. 45, S. 350 .

Ir. $\mathrm{Childe}, \mathrm{Ch}$ arles $\mathrm{P}$., A case of successful resection of nine and a half feet of small intestine for gangrene. The British Medical Journal I907, Vol. II, Oct. 5, S. $89 \mathrm{r}$.

12. De $\mathrm{n} \mathrm{k}$, W o l $\mathrm{g}$ a $\mathrm{ng}$, Ausgedehnte Darmresektion mit Ausgang in Heilung. Wiener klin. Wochenschr. I907, Nr. 52, S. 1649.

13. Derselbe, Erwiderung auf die Arbeit von Prof. Albu über Versuche über Ausschaltung von Dünn- und Dickdarm. Mitteilungen aus den Grenzgebieten der Medizin und Chirurgie 1909, Bd. 20, S. 215.

14. De rs e I be , Über die Prognose ausgedehnter Dünndarmresektionen. Ebendaselbst $1911, \mathrm{Bd} .22, \mathrm{~S}$. 146 .

I5. Di liberti-Herbin, Sulle estese resezioni dell' intestino tenue. Gazzetta Medica Italiana I903, Nr. 4I. Referiert in Zentralbl. f. Chir. 1904, Nr. 18. S. 572 .

16. D r e e s m a n n, Über größere Darmresektionen. Berliner klin. Wochenschr. I $899, \mathrm{Nr}, \mathrm{i} 6, \mathrm{~S} .337$.

17. Dreike, Pa u 1, Ein Beitrag zur Kenntnis der Länge des menschlichen Darmkanals. Deutsche Zeitschr. f. Chir. 1895, Bd. 40, S. 43. 
Experiment. u. literar. Studien über d. ausgedehnten Dünndarmoperationen.

18. Enochin, Ein Fall von umfassender Resektion des Dünndarms, Russ. chirurg. Archiv 1909, Buch 3, zitiert nach E. Istomin.

19. Erlanger und $\mathrm{Hew}$ e $\mathrm{t} t$. A study of the metabolism in dogs with shortened small intestines. The American Journal of Physiology I902, Vol. VI, S. I

20. Evans und Brenizer, Notes on the resection of large portions of the small intestine. Bulletin of the Johns Hopkins Hosp.tal 1907, Vol. 18, Nr. 20r, S. 477 .

2. Fa n tino, Giustepe, Contributo allo studio delle estese resezioni intestinali. Gazzetta medica di Torino $1 \$ 96, \mathrm{Nr}$. Io. Referiert in Zentralblatt für Chirurgie I $896, \mathrm{Nr} .25, \mathrm{~S}, 6$ I4.

22. Derselbe, Nuovo contributo allo studio delle estese resezioni intestinali, Riforma medica I902, Vol. IV, S. 736, 748 und $75^{\circ}$.

23. Fischer, A., Geheilter Fall von ausgedehnter Darmresektion. Orvosi Hetilap 1905, Nr. 37. Referiert in Zentralblatt für die Crenzgebiete der Medizin und Chirurgie I (x), Bd.9. Ar. 2, S. 70.

24. Flint, Jose ph Marshal1, Compensatory Hypertrophy of the Small Intestine Following Resection of Large Portions of the Jejunum and Ileum. Yale Medical Journal igro, Vol. 16, Nr. 6, S. $28,3$.

25. Fran $z$, Herniologische Beobachtungen mit besonderer Berücksichtigung der Radikaloperationen, mitgeteilt aus der Königlich-chirurgischen Universitätsklinik des Herrn Professor v. Eiselsberg. Deutsche Zeitschrift für Chirurgie 1899 , Bd. 51, S. 78 und 98 .

26. Friedrich, P. L., Uber den Nutzen der funktionellen Ausschaltung grober Darmabschnitte bei septischer Peritonitis. Medizinische Klinik r 905 , Nr. 2, S. 25.

27. Ghedini. An nibale, Contributo alle estese resezioni ed esclusioni dell' intestino. La Clinica chirurgica I905. Anno I3, S. I217.

28. Göbe11, K u dolf, Erfolgreiche Resektion von $3 \mathrm{~m}$ Ileum wegen Volvulus und innerer Einklemmung. Deutsche Zeitschr. f. Chir. I905, Bd. 77. S. 608 .

29. Harris, George R., Rescction of nearly eight feet of gangrenous intestine-Recovery. Medical Record I g(x)2, Vol. 62, S. 563.

30. Is t o m in, E., Zur Kenntnis der Cysten und festen Geschwülste des Mesenteriums. Russische medizinische Rundschau. 1910, 8. Jahrgang, S. 329 und 373.

31. Ka rlow, Resektion af $2 \mathrm{~m} 15 \mathrm{~cm}$ tarm. Hälsa. Hygiea, N. F., I906, S. 267. Referiert in Zentralblatt für dic Grenzgebiete der Medizin und Chirurgie 1907 , Bd. Io, Nr. I 3, S. 5 I 5 .

32. Ko e be r lé, Résection de deux mètres d'intestin grêle. Bulletin de l'académie de médecine 1881,2 . Série, Tome ro, S. I 28.

33. Kopfstein, W., Beitrag zu ausgedehnten Darmresektionen. Revue der böhm. Medizin Igog, Heft 4. Referiert in Wiener Mediz. Blätter I9IO, Nr. z. S. 16 . 
34. Kukula, Uber ausgedehnte Darmresektionen. Arch. f. klin. Chir. I900, Bd. 60 , S. 887 .

35. L a u e n te in, Carl, Ist es möglich, die ,zwei Darmschlingen im eingeklemmten Bruch" zu diagnostizieren? Zugleich ein neuer Beitrag zu der Pathologie der ,Hernie en W" resp. der ,retrograden Darminkarzeration“. Deutsche Zeitschr. f. Chir. I909, Bd. I00, S. 169.

36. L a u wers, Des résections étendues de l'intestin grêle. Journ. de chir. et ann. de la soc. belge de chir. I901, Nr. 12. Referiert in Zentralblatt f. Chir. 1902, Nr. 2 I, S. 574 .

37. Lexer, E., Operation eines Mesenterialfibromes mit ausgedehnter Resektion des Dünndarmes. Berl. klin. Wochenschr. I900, Nr. I, S. 4.

38. Lieblein, Viktor, Für die Prognose der ausgedehnten Dünndarmresektionen. Verein deutscher Arzte zu Prag. Sitzung vom 2. Dez. Ig1o. Berl. klin. Wochenschr. I 9 I I, Nr. 3, S. I 5 I.

39. Derse 1 be, Einige Bemerkungen zur Frage der Prognose der ausgedehnten Dünndarmresektionen. Mitteilungen aus den Grenzgebicten der Medizin und Chirurgie I9I I, Bd. 23. S. I.

40. London, E. S. und W. D m it riew, Zum Chemismus der Verdaung und Resorption im tierischen Körper. XXXIX. Mitteilung. Über die Verdauung und Resorption nach Darmausschaltungen. Zeitschrift für physiologische Chemie 1910, Bd. 65, S. 213.

4I. L ore n z, H a n s, K. K. Gesellschaft der Arzte in Wien. Sitzung II. Mai 1906. Wiener klinische Wochenschr. I906, Nr. 20, S. 610.

42. Mitchell, A. B., Diskussion. The British medical Association, Section of surgery. July 28.1909 at Belfast. Medical Record Igo9, Vol. 76. Nr. 8, S. 330 .

43. M i ya ke, H., Uber ausgedehnte Darmresektion mit einer kurzen Bemerkung über die normale Länge des Jejuno-Ileum bei dem Lebenden. Archiv f. klin. Chir. 1910, Bd. 93, S. 768.

44. Monari, Umberto, Experimentelle Untersuchungen über die Abtragung des Magens und cles Dünndarms be $m$ Hunde. Beiträge zur klin. Chir. I 896, Bd. I6, S. 479 .

45. M on profit, Résection de trois mètres dix centimètres d'intestin dans une cure radicale de hernie volumineuse. Congrès français de chirurgie. Treizième session 1899 , S. 427 .

46. Nag a n o, J., Zur Frage ausgedehnter Dünndarmresektion. Beiträge zur klin. Chir. I903, Bd. 38, S. 431 .

47. $\mathrm{Nicola}, \mathrm{B}$ a i a, Resezione di m. 3,50 di intestino tenue per ernia ombellicale strozzata. Studium Nr. 3, 30. Marzo 1910. Referiert in La Clinica chirurgica I9I0, Anno i8, S. I 347.

48. $\mathrm{N}$ ig r is o I i, B., Sulle resezioni estese dell'intestino. Archivio et atti della società italiana di chirurgia 1902, Anno I7, S L.

49. Pa rk, R os we 11, Successful removal of $265 \mathrm{~cm}$ of gangrenous intestine. Archives internationales de chirurgie 1903, Vol. I, S. 106. 
50. P a u che t. V., Résection de 4 mètres d'intestin grêle. Gaz. méd. de Picardie, nov. 1905 . Referiert in Gazette des hôpitaux I905. Anné 78, Nr. I 39 , S. 1667 .

51. Pa yr, Erwin, thber ausgedehnte Darmresektionen. Archiv f. klin. Chir. I902, Bd. 67. S. I81.

52. Pok ot il o, Ein Fall von ausgedehnter Dünndarmresektion. Chirurgia I g(x), Nr. I 46 , S. 169. Referiert in Jahresbericht über die Fortschritte anf dem Gebiete der Chir. Iox)9, Jahrgang I5, S. 726.

53. $\mathrm{R}$ a u be $\mathrm{r}$. A., Lehrbuch der Anatomic des Menschen. V. Aufl., Bd. I. Leip)zig 1897 , S. 580 .

54. R i va-R o c c i, La funzione intestinale dopo una resezione estesa del tenue. Gazzetta medica di Torino 1896 , Nr. 7. Referiert in Zentralbl. f. Chir. I896, Nr. 25, S. 614.

53. Rothc, Hermann, Beitrag zur Statistik der incarcerierten Hernien. Beiträge zur klin. Chir. I902, Bd. 33 , S. 184.

56. R u g g i, Cri us e p pe, Storia relativa ad un fanciullo d'anni otto al quale furono asportati con successo $\mathrm{m} .3 \cdot 30 \mathrm{~d}$ 'intestino tenue. Il Policlinico. Sezione chirurgica is 96 , Volume 3, S. 49 .

57. Rusch ha pt, Walther, Cher ausgedehnte Darmresektionen. Inaug.Diss. Bonn igur.

5*. Sa p p c y. Traité d'Anatomic descriptive. IV. I874. Zitiert nach Dreike.

sy. Schlat $\mathrm{ter}, \mathrm{C}$ arl. The process of ligestion after resection of about $-\mathrm{ix}$ fect of the small intestine. The Lancet. I $y(x)$ Jan. 27. S. 2 i 7.

o. Derselbe, Über die Darmfunktion nach ausgedchnten Darmresektionen. Beitr. zur klin. Chir. Jqu, Bd. 49, S. I.

or. Senn, Nicholas, An experimental contribution to intestinal surgery with spezial reference to the treatment of intestinal obstruction. Annals of surgery 1888 , Vol. 7, S. 99 .

6. Shepherd, Francis J.. Successful removal of an enormous mesenteric tumour and nearly eight feet of intestine. The British Medical Journal I897, Oct. 9, S. $g^{2}(6)$

63. Spassokukozkaja, Ein Fall von augedehnter Dündarmrestion. Russki Wratsch Igod, Nr. 2. Ref. in Zentralbl. f. Chir. Igoo, Nr. 24, S. 687.

64. St a e h lin. Ed ward, Resection of ten feet, two inches of small intestine, with recovery. Annals of surgery 1907 , Vol, 45 , S. 49.

65. Stolz. Ausgedehntc Darmresektion wegen Gangrän. Unterclsäßischer Arzteverein in Straßburg i. E. Sitzung am 19. Dez. Igo8. Deutsche Mediz. Wochenschr. 19(9), Nr. 16, S. 744.

66. Dersclbe, Spätresultat einer ausgedehnten Dünndarmrescktion. Berl. klin. Wochenschr. I9J0, Nr. 36, S. 1685.

67. S t or p, Über die Zulässigkeit ausgedehnter Dünndarmresektionen. Deutschte Zeitschr. f. Chir. I go\%, Bd. 87, S. $3^{13}$.

(8). Tak a yas u, Experimentelle Intersuchungen über die Grenzen der $Z_{11}$ lässigkeit ausgedehnter Darmausschaltungen. Mitt. der mediz. Gesellsch. zu Tokio 1909, Bd. 23, S. 127 (Japanisch). 
69. Th o n, Über Knotenbildung des Dünndarms. Mediz, Gesellsch. in Giessen. Sitzung am I5. Dez. 1908. Deutsche Mediz. Wochenschr. I909, Nr. 16, S. 742. jo. Trzebicky, Rudolf, Über die Grenzen der Zulässigkeit der Dünndarmresektion. Archiv f. klin. Chir. I 894, Bd. 48, S. 54.

71. Vita $1 \mathrm{i}$, Ricerche sulla funzione intestinale e sul ricambio materiale in un caso di estesissima resezione del tenue. Rivista critica di clinica medica 1902, Nr. II-I3. Referiert in Zentralbl. f. die Grenzgeb.ete der Medizin und Chir. 1903 , Bd. 6, Nr. i , S. 440.

72. We relius, Axel, Successful resection of twelve feet and two inches of the ileum in case of criminal abortion. The Journal of the American Medical Association 1907 , Vol. 48 , S. 945 .

73. $\mathrm{Zusch}$, Stoffwechsel nach ausgedehnter Dünndarmresektion. Arztlicher Verein in Danzig. Sitzung am Io. Dez. I908. Deutsche med. Wochenschr. I909, Nr. 16, S. 739. 